\title{
Hochschild and Cyclic Homology of Quantum Groups
}

\author{
Ping Feng ${ }^{1, \star}$ and Boris Tsygan ${ }^{2, \star \star}$ \\ ${ }^{1}$ Department of Physics, Harvard University, Cambridge, MA 02138, USA \\ 2 Department of Mathematics, Harvard University, Cambridge, MA 02138, USA
}

Received October 23, 1990; in revised form February 20, 1991

\begin{abstract}
For an arbitrary complex linear semisimple Lie group $G$, we consider Hopf algebras of the deformations of the formal and algebraic functions on $G$. The Hochschild and cyclic homology of these Hopf algebras are computed when the value of the deformation parameter is generic.
\end{abstract}

\section{Table of Contents}

1. Introduction . . . . . . . . . . . . . . . . . . 481

2. Definitions of Hochschild and Cyclic Homology . . . . . . . . 484

3. Quantum Groups . . . . . . . . . . . . . . . . . . . 488

4. A Spectral Sequence . . . . . . . . . . . . . . . . . . 493

5. The Lie Algebra Homology . . . . . . . . . . . . . . . 500

5.1. Formal Functions . . . . . . . . . . . . . . . . . 501

5.2. Algebraic Functions . . . . . . . . . . . . . . . . 502

6. Hochschild and Cyclic Homology of Quantum Groups . . . . . 505

6.1. Quantum Formal Groups . . . . . . . . . . . . . . 506

6.2. Quantum Algebraic Groups . . . . . . . . . . . . . 506

Appendix A. Notations about Calculus on Lie Groups . . . . . . . 515

Appendix B. Example $G=S L(2, \mathbf{C})$. . . . . . . . . . . . . . 516

\section{Introduction}

According to Gelfand, the category of locally compact topological spaces is equivalent to the category of commutative $C^{*}$-algebras. Also, the category of affine

The main result of this paper was announced at the AMS meeting at University Park in April 1990

$\star$ Supported by Department of Energy under Grant DE-FG02-88ER25062

$\star \star$ Supported by Harvard Prize Fellowship. Address after July, 1990: Department of Mathematics, Pennsylvania State University, University Park, PA 16802, USA 
schemes of finite type over a field is equivalent to the category of finitely generated algebras over the field. Since the study of commutative algebras is the same as that of spaces, it is reasonable to interpret the study of non-commutative algebras as studying some "non-commutative spaces," and try to generalize the methods for spaces to be applicable to algebras. Cyclic (co)homology [8, 44, 14, 27] generalizes the de Rham cohomology to the non-commutative case, and it is sometimes referred to as non-commutative differential geometry.

In this paper we compute the Hochschild and cyclic homology of quantum groups, i.e., the quantized rings of functions on semi-simple Lie groups. This is interesting in several aspects. First it enriches the study of non-commutative differential geometry. Even though cyclic homology had been invented as generalized differential geometry and has found many applications, there is still a lack of interesting examples. Previously the only quantum spaces (in the spirit of Manin [31]) whose cyclic homologies were known were non-commutative tori $[8,43]$, quantum affine spaces [32] and $S U_{h}(2)$ [33]. However quantum groups $[11,21]$ present some of the most interesting examples of non-commutative spaces. Quantized universal enveloping algebras, which are more or less dual to what we are studying in this paper, were discovered in the course of studying integrable systems. They have been shown to have intimate relations with many other areas of mathematics and physics, e.g., link invariants [23], conformal field theories $[1,34,30]$. But most of what we know about quantized universal enveloping algebras concerns their representations, and their relations with other fields are simply observed without being explained. The knowledge of the structure of quantum groups is likely necessary to understand all these. A study of non-commutative differential geometry will provide much needed insight into the geometrical and topological properties of quantum groups.

The main phenomenon concerning the Hochschild and cyclic homology of quantum groups is the following. As is well-known, any deformation of the multiplicative structure of the algebra of functions on a manifold gives rise to a Poisson structure on the manifold; the symplectic leaves of this Poisson structure are in many senses good analogues of the "points of the non-commutative spectrum" of the deformed algebra. It has been established in several works that the non-commutative algebraic geometry of quantum spaces is very close to the geometry of "the space of symplectic leaves," i.e., the geometry of the quasiclassical limit of the quantum spaces, if the value of the deformation parameter is generic. We should mention the work of S. Levendorski, Y. Soibelman and L. Vaksman $[26,41,42]$ which asserts that the set of unitary representations of the quantized ring of functions on a compact semisimple Lie group is naturally isomorphic to the set of symplectic leaves of the Poisson structure. Also the approach to non-commutative algebraic geometry suggested by A. Rosenberg seems to give analogous results (in computed examples) [36]. Recall that the Hochschild homology of the algebra of algebraic functions on a non-singular affine variety is isomorphic to the space of differential forms on the variety. Thus the Hochschild homology of quantum groups may be viewed as the space of quantized differential forms. Our main result is that when the value of the deformation parameter is generic this Hochschild homology is equal to the space of "forms" on the "space of symplectic leaves" of the Poisson structure. The structure of symplectic leaves is of course well-known. When $G$ is a compact Lie group with 
maximal torus $T$, the Poisson structure on $G$ descends to the one on $G / T$, and its symplectic leaves are the Bruhat cells [39]. When $G$ is complex semi-simple the answer is similar.

Note that while the Hochschild homological dimension of $\mathbf{C}[G]$ is of course the dimension of the group, the analogous dimension for the quantized $\mathbf{C}[G]$ is the rank of the group. As has been mentioned by A. Connes, this phenomenon might give a more appropriate version of his approach to the various models of filed theory as "non-commutative Yang-Mills models" because when the Hochschild homological dimension drops, we get rid of various divergences [9].

Note also that the Hochschild and cyclic homology of the rings which are deformations of the commutative rings is a very interesting subject. In all reasonable cases the same phenomenon takes place: the non-commutative geometry is isomorphic to the geometry of the quasi-classical limit, for example, the (pseudo)differential operators on a manifold $[2,4,46]$, universal enveloping algebra $[15,22]$, and non-commutative tori $[8,43]$. But the explicit form of this isomorphism is highly non-trivial. For the ring of differential operators on a manifold, this explicit isomorphism implies the Riemann-Roch theorem; for the ring of symbols of pseudo-differential operators, the analogous statement is very likely to imply the index theorem $[16,46]$. We hope that these statements are the partial cases of a conjectural generalized Riemann-Roch theorem which measures the "non-triviality" of the isomorphism of Hochschild and cyclic homology of the quasi-classical limit and that of the quantum limit. In this respect, the quantum groups (or rather the quantum homogeneous spaces) may provide another non-trivial partial case.

The paper is organized as follows. In Sect. 2 we recall the definition and basic properties of Hochschild and cyclic homology. Here we give a new complex for computing the Hochschild homology of Hopf algebras. Section 3 describes the quantum groups we are going to study. The rest of the paper is mainly devoted to the computation of the Hochschild homology of quantum groups. In Sect. 4 we introduce a spectral sequence, which converges to Hochschild homology of quantum groups. Its $E^{2}$ term is the Brylinski homology of the group regarded as a Poisson manifold. It is then identified with Lie algebra homology of $\mathbf{g}^{*}$, the dual of the Lie algebra $g$ of the corresponding group $G$ of the quantum group, with coefficient in the functions on $G$. The geometrical meaning of the action is given, and it is shown that it is related to the standard Lie algebra action on some very simple induced representations. We compute this Lie algebra homology for both formal and algebraic functions in Sect. 5. We then show in Sect. 6 that for both formal and algebraic cases the spectral sequence of Sect. 4 is degenerate at $E^{2}$. This means the quantum limit is determined by the quasiclassical limit. Thus the Lie algebra homology actually yields the Hochschild homology. After this the cyclic homology is readily obtained. In Appendix A we give some of our conventions about the calculus on Lie groups. Appendix B contains various explicit calculations for $G=S L(2, \mathbf{C})$.

We are grateful to J. L. Brylinski, A. Connes, V. G. Drinfeld, B. Enriquez, E. Getzler, M. Goulian, N. Yu. Reshetikhin for helpful discussions. We are especially indebted to W. Schmid for consultation on the n-homology of Harish-Chandra modures. In our first draft there was a gap in the proof of the degeneracy of a spectral sequence. We wish to express our deep gratitude to J. Block and E. Getzler 
for pointing that out to us. P. F. would like to thank A. Jaffe for his encouragement and support.

\section{Definitions of Hochschild and Cyclic Homology}

Here we recall the definitions and basic properties of Hochschild and cyclic homology $[8,44,14,27]$. Let $A$ be an associative algebra over a commutative unital ring $k, k \subset A$. In this paper $k=\mathbb{C}$, or $\mathbb{C}((h))$, the ring of Laurent series. The Hochschild homology of $A$, denoted by $H_{*}(A)$, is defined to be the homology of the following complex

$$
0 \longleftarrow C_{0}(A) \stackrel{b}{\longleftarrow} C_{1}(A) \stackrel{b}{\longleftarrow} C_{2}(A) \stackrel{b}{\longleftarrow} \cdots,
$$

where $C_{n}(A)=A^{\otimes n+1}$, and $b: C_{n}(A) \rightarrow C_{n-1}(A)$ such that

$$
\begin{aligned}
b\left(a_{0} \otimes a_{1} \otimes \cdots \otimes a_{n}\right)= & \sum_{i=0}^{n-1}(-1)^{i} a_{0} \otimes a_{1} \otimes \cdots \otimes a_{i} a_{i+1} \otimes \cdots \otimes a_{n} \\
& +(-1)^{n} a_{n} a_{0} \otimes a_{1} \otimes \cdots \otimes a_{n-1} .
\end{aligned}
$$

The cyclic homology of $A$, denoted by $H C_{*}(A)$, is defined to be the total homology of the following double complex

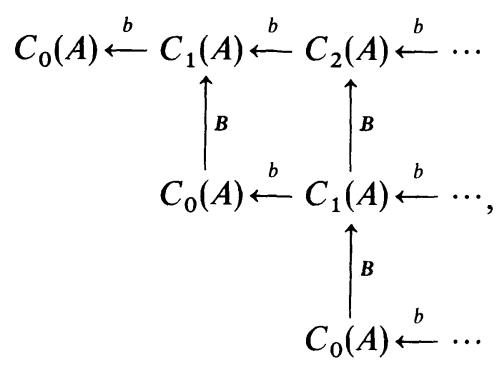

where $B: C_{n}(A) \rightarrow C_{n+1}(A)$ such that

$$
\begin{aligned}
B\left(a_{0} \otimes a_{1} \otimes \cdots \otimes a_{n}\right)= & \sum_{i=0}^{n}(-1)^{n i}\left(1 \otimes a_{i} \otimes \cdots \otimes a_{n} \otimes a_{0} \otimes \cdots \otimes a_{i-1}\right. \\
& \left.+(-1)^{n} a_{i} \otimes \cdots \otimes a_{n} \otimes a_{0} \otimes \cdots \otimes a_{i-1} \otimes 1\right) .
\end{aligned}
$$

If $k=\mathbf{C}((h))$ and $A$ is $h$-adically complete, then by the tensor product we will always mean $h$-adically completed tensor product.

Let $X$ be a non-singular affine variety, $A=\mathrm{C}[X], \Omega^{n}(X)$ the $n$-forms on $X$, $H_{\mathrm{DR}}^{n}(X)$ the $n^{\text {th }}$ de Rham cohomology of $X$. The following result is well-known.

\section{Lemma (2.1).}

1) (Hochschild-Kostant-Rosenberg) $H H_{n}(A)=\Omega^{n}(X)$,

2) $H C_{n}(A)=\Omega^{n}(X) / d \Omega^{n-1}(X) \oplus H_{\mathrm{DR}}^{n-2}(X) \oplus H_{\mathrm{DR}}^{n-4} \otimes \cdots$.

This shows that in the commutative case the cyclic homology measures the de Rham cohomology of the space, thus the name "non-commutative differential geometry."

We are especially interested in certain Hopf algebras. Let us recall 
Definition (2.2). A Hopf algebra A over $k$ is a $k$-bimodule A together with morphisms $m, i, \Delta, \varepsilon, S$,

$$
\begin{gathered}
A \otimes A \stackrel{m}{\rightarrow} A \stackrel{\Delta}{\rightarrow} A \otimes A, \\
A \stackrel{\varepsilon}{\longrightarrow} k \stackrel{\iota}{\longrightarrow} A, \\
A \stackrel{s}{\longrightarrow} A,
\end{gathered}
$$

such that they satisfy the following axioms:

1. Associativity:

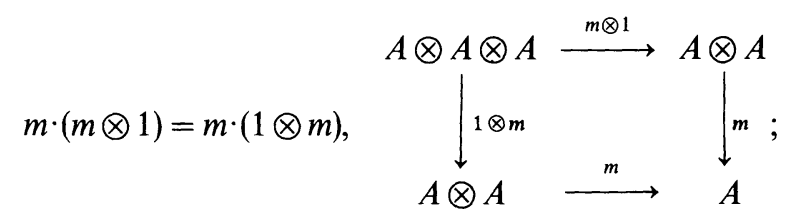

2. Unit:

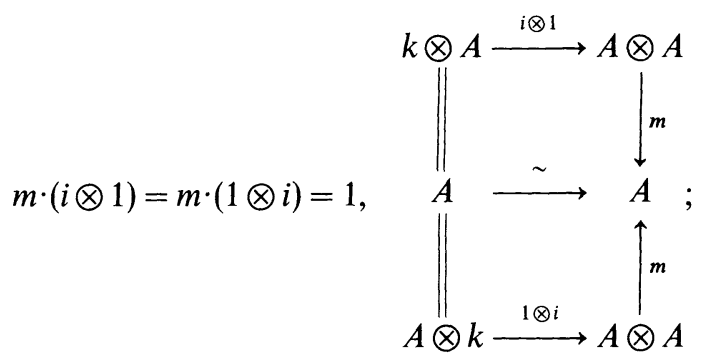

3. Coassociativity:

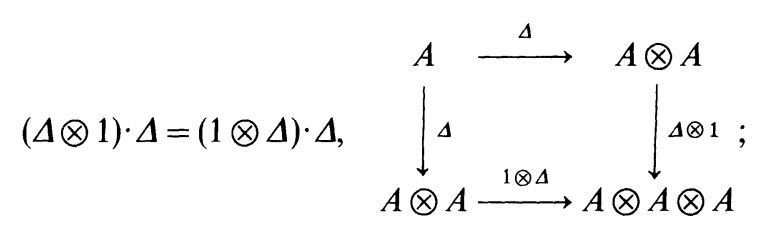

4. Counit:

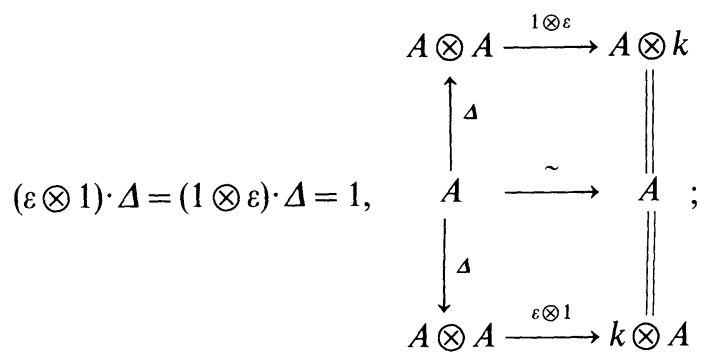


5. Connection Axiom:

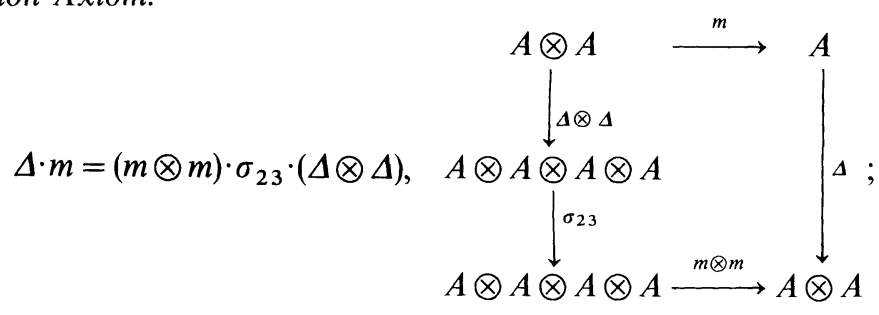

where $\sigma_{23}$ means the permutation between the second and the third variables;

6. Antipode:

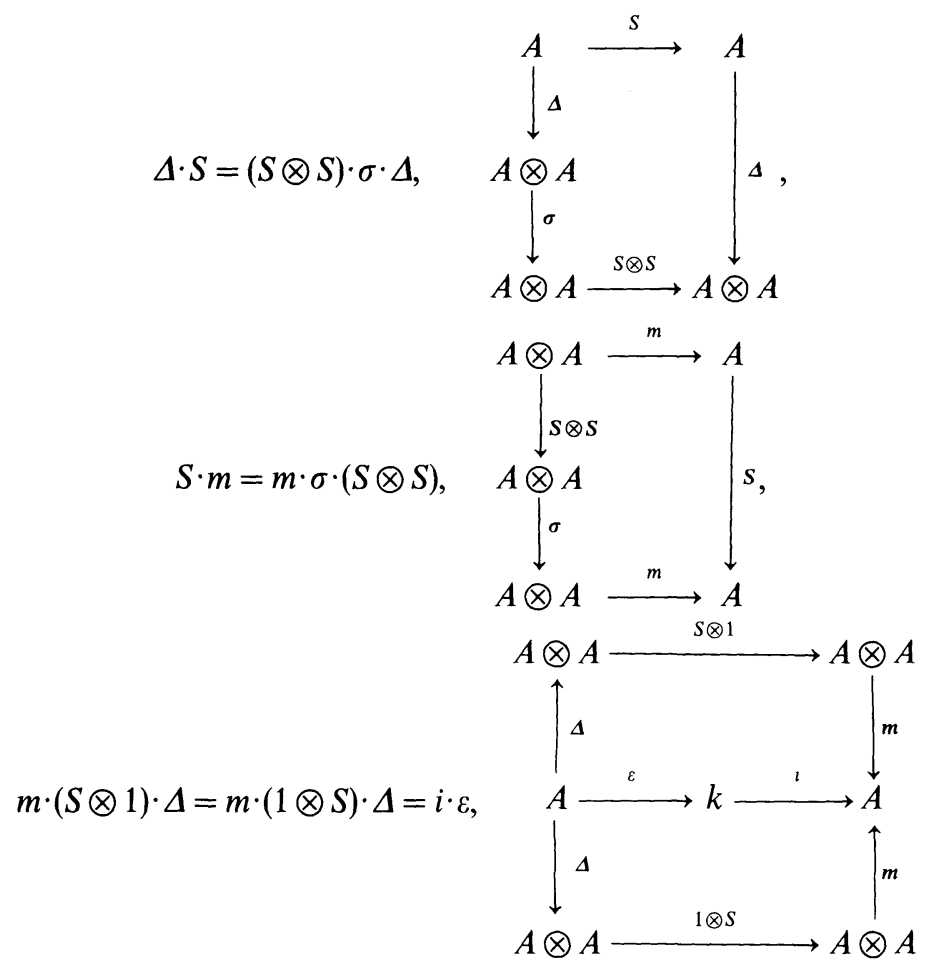

We will call $m, i, \Delta, \varepsilon, S$, respectively, the product, unit, coproduct, counit, antipode of Hopf algebra A. We usually omit the symbol $m, i$ when there is no confusion.

Example (2.3). Let $G$ be complex algebraic group, $\mathbf{g}$ its Lie algebra, $U(\mathbf{g})$ the universal enveloping algebra, $\mathbf{C}[G]$ the algebraic functions on $G$.

$U(g)$ is made into a Hopf algebra by

$$
\begin{aligned}
\forall x \in \mathbf{g}, \quad \Delta(x) & =1 \otimes x+x \otimes 1, \\
\varepsilon(x) & =0, \\
S(x) & =-x .
\end{aligned}
$$


$\mathbf{C}[G]$ is made into a Hopf algebra by

$$
\begin{aligned}
\forall f \in \mathbf{C}[G], \quad g, h \in G, \quad(\Delta(f))(g, h) & =f(g h), \\
(\varepsilon(f))(g) & =f(e), \\
(S(f))(g) & =f\left(g^{-1}\right),
\end{aligned}
$$

where $e$ is the identity of $G$.

The formal functions on $G$ in the vicinity of identity, $\mathbf{C}^{f}(G)$, can be viewed as a Hopf algebra dual to the universal enveloping algebra $U(\mathbf{g})$.

We now give a new complex for Hochschild homology of Hopf algebras. ${ }^{1}$ Let $m_{r}, m_{l}$ denote respectively the natural right, left multiplications of $A$ on $A$, i.e., $m_{r}(a) b=b a, m_{l}(a) b=a b$. Define a right action of $A$ on $A$ by

$$
\rho(a)=\left(m_{r} \otimes m_{l}\right)(1 \otimes S) \Delta(a) .
$$

This obviously defines a right action since $\Delta$ is algebra homomorphism and $S$ is antihomomorphism. To be more specific,

$$
\rho(a) x=\sum_{i} \tilde{a}_{i}^{s} x a_{i}
$$

where $\Delta(a)=\sum_{i} a_{i} \otimes \tilde{a}_{i}$, and $\tilde{a}^{s}=S(\tilde{a})$.

Consider the following complex $(C .(A), d)$ :

$$
0 \longleftarrow C_{0}(A) \stackrel{d}{\longleftarrow} C_{1}(A) \stackrel{d}{\longleftarrow} C_{2}(A) \stackrel{d}{\longleftarrow} \cdots,
$$

where $d: C_{n}(A) \rightarrow C_{n-1}(A)$, such that

$$
\begin{aligned}
d\left(a_{0} \otimes a_{1} \otimes \cdots \otimes a_{n}\right)= & \rho\left(a_{1}\right) a_{0} \otimes a_{2} \otimes \cdots \otimes a_{n} \\
& +\sum_{i=1}^{n-1}(-1)^{i} a_{0} \otimes \cdots \otimes a_{i} a_{i+1} \otimes \cdots \otimes a_{n} \\
& +(-1)^{n} \varepsilon\left(a_{n}\right) a_{0} \otimes a_{1} \otimes \cdots \otimes a_{n-1} .
\end{aligned}
$$

It is straightforward to check that $d^{2}=0$, thus it indeed defines a complex. Note that in this paper we also use $d$ to denote the exterior derivative on $G$. We hope there is no confusion as to which $d$ we are referring to.

Define $\xi: C_{n}(A) \rightarrow C_{n}(A)$ by

$$
\xi(x \otimes a \otimes b \otimes \cdots \otimes c)=\sum_{i, j, \ldots, k} \tilde{c}_{\ddot{k}}^{s} \cdots \tilde{b}_{j}^{s} \tilde{a}_{i}^{s} x \otimes a_{i} \otimes b_{j} \otimes \cdots \otimes c_{k},
$$

where $\Delta(a)=\sum_{i} a_{i} \otimes \tilde{a}_{i}$, etc.

1 This complex might have been known to other people, but we are unable to locate it in the literature other than [13] 


\section{Proposition (2.4).}

1) $\xi: C_{n}(A) \stackrel{\sim}{\rightarrow} C_{n}(A)$

2) $\xi d=b \xi$, i.e., the following diagram is commutative,

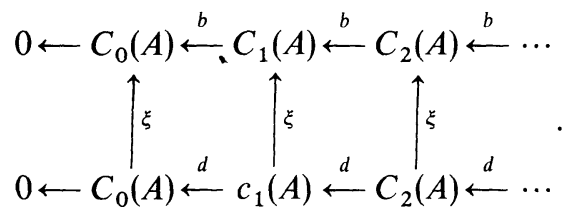

Proof. 1) We define $\xi^{\prime}: C_{n}(A) \rightarrow C_{n}(A)$, by

$$
\xi^{\prime}(x \otimes a \otimes b \otimes \cdots \otimes c)=\sum_{i, j \ldots, k} \tilde{a}_{i} \tilde{b}_{j} \cdots \tilde{c}_{k} x \otimes a_{i} \otimes b_{j} \otimes \cdots \otimes c_{k} .
$$

We claim that $\xi^{\prime} \xi^{\prime}=\xi^{\prime} \cdot \xi=1$. Let us check it for $n=1$, the general case can be shown similarly. Denote $\Delta(a)=\sum_{i} A_{i} \otimes B_{i}, \Delta^{2}(a)=(1 \otimes \Delta)(a)=(\Delta \otimes 1)(a)=\sum_{j} a_{j} \otimes$ $b_{j} \otimes c_{j}$, then,

$$
\begin{aligned}
\xi \cdot \xi^{\prime}(x \otimes a) & =\xi\left(\sum_{i} B_{i} x \otimes A_{i}\right)=\sum_{j} b_{j}^{s} c_{j} x \otimes a_{j} \\
& =\sum_{j} \varepsilon\left(B_{j}\right) x \otimes A_{j}=x \otimes \sum_{j} \varepsilon\left(B_{j}\right) A_{j}=x \otimes a,
\end{aligned}
$$

where we have used $m \cdot(S \otimes 1) \cdot \Delta=\varepsilon$, and $(\varepsilon \otimes 1) \cdot \Delta=1$. Thus $\xi \cdot \xi^{\prime}=1$. Similarly, $\xi^{\prime} \cdot \xi=1$.

2) Again we only check the $n=1$ case.

$$
\xi \cdot d(x \otimes a)=\xi\left(\sum_{j} B_{j}^{s} x A_{j}-\varepsilon(a) x\right)=\sum_{j} B_{j}^{s} x A_{j}-\varepsilon(a) x,
$$

and

$$
b \cdot \xi(x \otimes a)=b\left(\sum_{j} B_{j}^{s} x \otimes A_{j}\right)=\sum_{j} B_{j}^{s} x A_{j}-\left(\sum_{j} A_{j} B_{j}^{s}\right) x=\sum_{j} B_{j}^{s} x A_{j}-\varepsilon(a) x .
$$

We get $\xi \cdot d=d \cdot \xi$.

Note that $(2.16,2.17)$ is the standard complex for the functor Tor $_{*}$. We have Corollary (2.5).

$$
H H_{*}(A)=H_{*}(C \cdot(A), d)=\operatorname{Tor}_{*}^{A}(A, k),
$$

where $A$ is considered as a right $A$-module via $\rho$ defined in (2.15), and $k$ is considered as a left $A$-module via the counit $\varepsilon$.

It is this new complex that we are going to use for computing the Hochschild homology of quantum groups. The new functorial interpretation will be helpful later in Sect. 6.

\section{Quantum Groups}

By quantum group we mean the Hopf algebra (over $\mathbf{C}[[h]]$ ) of the formal deformation of the functions on a group. We will start with a connected, complex, semi-simple Lie group $G$ and consider the formal deformation of formal functions 
$\mathbf{C}^{f}(G)$ and algebraic functions $\mathbf{C}[G]$, resulting in respectively quantum formal group $\mathbf{C}_{h}^{f}(G)$ and quantum algebraic group $\mathbf{C}_{h}[G]$. The data we need for the formal deformation is a Poisson Lie group structure on $G$, which we refer to as the quasiclassical limit of quantum groups. Dually this is equivalent to specifying a Lie bialgebra structure on $\mathbf{g}$, the formal deformation of which results in the quantized universal enveloping algebra $U_{h}(\mathbf{g})$.

We start with a description of the quasiclassical limit of quantum groups. Much of what we state here can be found in $[12,28]$.

Definition (3.1). A Lie group $G$ is called a Poisson Lie Group if it is a Poisson manifold such that the group multiplication $G \times G \rightarrow G$ is a Poisson map, where $G \times G$ is equipped with the product Poisson structure. The Poisson structure is specified by a bivector field $\pi$, the Poisson tensor,

$$
\{f, g\}=\langle\pi, d f \wedge d g\rangle, \quad \forall f, g \in C^{\infty}(M) .
$$

A map $\phi: M \rightarrow N$ between two Poisson manifolds is a Poisson map if $\left\{\phi^{*} f, \phi^{*} g\right\}_{M}=\{f, g\}_{N}$.

Definition (3.2). Let $\mathbf{g}$ be a Lie algebra over $\mathbf{C}, \mathbf{g}^{*}$ its dual. We say that (g) is a Lie bialgebra if there is a Lie algebra structure on $\mathbf{g}^{*}$ such the map $\delta: \mathbf{g} \rightarrow \wedge^{2} \mathbf{g}$ dual to the Lie bracket $[]:, \wedge^{2} \mathbf{g}^{*} \rightarrow \mathbf{g}^{*}$ is a 1-cocycle with the adjoint action of $\mathbf{g}$ on $\wedge^{2} \mathbf{g}$.

The following theorem states the relations between Poisson Lie groups and Lie bialgebras.

Theorem (3.3). [11]

1) Let $G$ be connected and simply connected, then there is one to one correspondence between Poisson Lie group structures on $G$ and Lie bialgebra structures on $\mathbf{g}$;

2. Let $G$ be connected and semi-simple, then every Poisson Lie group structure on $G$ has the following form:

$$
\pi(g)=l_{g^{*}} R-r_{g^{*}} R,
$$

where $R \in \wedge^{2} \mathbf{g} \cong \wedge^{2} T G(e), g \in G, l_{g^{*}}$ and $r_{g^{*}}$ are push-forward maps of left and right translations, and $R$ is such that

$$
\begin{aligned}
\langle R, R\rangle:= & {\left[R^{12}, R^{13}\right]+\left[R^{12}, R^{23}\right]+\left[R^{13}, R^{23}\right] \in \wedge^{3} g } \\
& \text { is invariant under adjoint action of } \mathbf{g} .
\end{aligned}
$$

The corresponding Lie bialgebra $(\mathbf{g}, \delta)$ is given by

$$
\delta(x)=\operatorname{ad}_{x}(R), \quad \forall x \in \mathbf{g},
$$

where ad is adjoint action. Condition (3.3) ensures the dual map of (3.4) satisfies Jacobi identify.

3. For the Lie bialgebra $\left(\mathbf{g}, \mathbf{g}^{*}\right)$ there is a unique Lie algebra structure on the vector space $\mathbf{g} \oplus \mathbf{g}^{*}$ such that

(a) $\mathbf{g}, \mathbf{g}^{*}$ are Lie subalgebras,

(b) The symmetric bilinear form $\langle$, $\rangle$ on $\mathbf{g} \oplus \mathbf{g}^{*}$ given by

$$
\left\langle x_{1}+y_{1}, x_{2}+y_{2}\right\rangle=\left\langle x_{1}, y_{2}\right\rangle+\left\langle x_{2}, y_{1}\right\rangle, \quad \forall x_{1}, x_{2} \in \mathbf{g}, \text { and } y_{1}, y_{2} \in \mathbf{g}^{*}
$$

is invariant. 
The Lie algebra structure on $\mathbf{g} \oplus \mathbf{g}^{*}$ is given by

$$
[x, y]=-\operatorname{ad}_{x}^{*}(y)+\operatorname{ad}_{y}^{*}(x), \quad \forall x \in \mathbf{g}, y \in \mathbf{g}^{*},
$$

where $\mathrm{ad}^{*}$ is the coadjoint action. This Lie algebra will be denoted by $\mathbf{g} \bowtie \mathbf{g}^{*}$ as in [28]. (g, $\left.\mathbf{g}^{*}, \mathbf{g} \bowtie \mathbf{g}^{*}\right)$ is called Manin triple.

We now describe the Lie bialgebras we are going to study. First of all, a few words about notations.

$G$ is a connected, complex, semi-simple Lie group, $\mathbf{g}$ its Lie algebra, $K$ is a compact real form of $G$, with Lie algebra $\mathbf{k}, \tau_{0}$ the complex conjugation of $\mathbf{g}$ with respect to $\mathbf{k}, B($,$) the Killing form of \mathbf{g}$, $\mathbf{h}$ a Cartan subalgebra of $\mathbf{g}, \Phi$ the root system of $(\mathbf{g}, \mathbf{h}), \Phi^{+}$a choice of positive root system. Let $\mathbf{g}=\mathbf{h} \oplus\left(\bigoplus_{\alpha \in \Phi} \mathbf{g}^{\alpha}\right)$ be the root space decomposition of $\mathbf{g}$. For each $\alpha \in \Phi$ we may choose $E_{\alpha} \in \mathbf{g}^{\alpha}$, and $H_{\alpha} \in \mathbf{h}$, such that [19]

(a) $B\left(E_{\alpha}, E_{\beta}\right)=\delta_{\alpha,-\beta},\left[E_{\alpha}, E_{-\alpha}\right]=H_{\alpha}$;

(b) $B\left(H_{\alpha}, H\right)=\alpha(H), \forall H \in \mathbf{h}$;

(c) $\left[E_{\alpha}, E_{\beta}\right]=0$ if $\alpha+\beta \notin \Phi, \alpha+\beta \neq 0$;

(d) $\left[E_{\alpha}, E_{\beta}\right]=N_{\alpha, \beta} E_{\alpha+\beta}$ if $\alpha+\beta \in \Phi$, where $N_{\alpha, \beta}$ are non-zero real constants such that $N_{-\alpha,-\beta}=-N_{\alpha, \beta}$; also $N_{\alpha, \beta}=N_{\beta, \gamma}=N_{\gamma, \alpha}$, whenever $\alpha, \beta, \gamma \in \Phi$, $\alpha+\beta+\gamma=0$

(e) $\tau_{0}\left(E_{\alpha}\right)=-E_{-\alpha}, \tau_{0}\left(H_{\alpha}\right)=-H_{\alpha}$.

Consider the complexification of $\mathbf{g}, \mathbf{g}_{\mathbf{c}} \equiv \mathbf{C} \otimes_{\mathbf{R}} \mathbf{g} \cong \mathbf{g} \oplus \mathbf{g}$ as $\mathbf{R}$ vector space. We choose the complex structure $J$ on $\mathbf{g} \oplus \mathbf{g}$ to be such that $J(x, y)=\left(J_{0} x, \mathbf{J}_{0} y\right)$, where $J_{0}$ is the complex structure of $\mathbf{g}$. We identify $\mathbf{g}$ as a real form of $\mathbf{g}_{\mathbf{c}}: \mathbf{g} \subset \mathbf{g} \oplus \mathbf{g}$, $\mathbf{x} \mapsto\left(\mathbf{x}, \tau_{0} \mathbf{x}\right)$. The complex conjugation of $\mathbf{g}_{\mathbf{c}}$ with respect to $\mathbf{g}$ is $\sigma(x, y)=\left(\tau_{0} y, \tau_{0} x\right)$. Obviously $\mathbf{k} \oplus \mathbf{k} \subset \mathbf{g} \oplus \mathbf{g}$ is the compact real form of $\mathbf{g}_{\mathbf{c}}$, the corresponding complex conjugation is $\tau(x, y)=\left(\tau_{0} x, \tau_{0} y\right)$. The Cartan involution is $\theta=\tau \sigma=\sigma \tau$, $\theta(x, y)=(y, x)$. The Iwasawa decomposition of $\mathbf{g}_{\mathbf{c}}$ is $\mathbf{g}_{\mathbf{c}}=\mathbf{k}_{\mathbf{c}} \oplus \mathbf{a}_{\mathbf{c}} \oplus \mathbf{n}_{\mathbf{c}}$, where

$$
\begin{aligned}
& \mathbf{k}_{\mathbf{c}}=\{(x, x) \mid x \in \mathbf{g}\} \cong \mathbf{g}, \\
& \mathbf{a}_{\mathbf{c}}=\{(x,-x) \mid x \in \mathbf{h}\}, \\
& \mathbf{n}_{\mathbf{c}}=\left\{(x, y) \mid x \in \mathbf{n}_{-}, y \in \mathbf{n}_{+}\right\},
\end{aligned}
$$

and $\mathbf{n} \pm=\bigoplus_{\alpha>0} \mathbf{g}^{ \pm \alpha}$.

Let $\mathbf{t}_{\mathbf{c}}=\{(\mathbf{x}, \mathbf{x}) \mid \mathbf{x} \in \mathbf{h}\}$. Then $\mathbf{h}_{\mathbf{c}}=\mathbf{t}_{\mathbf{c}} \oplus \mathbf{a}_{\mathbf{c}}$ is a Cartan subalgebra of $\mathbf{g}_{\mathbf{c}}$. For every $\alpha \in \Phi(\mathbf{g}, \mathbf{h})$, we have $\alpha^{1}, \alpha^{2} \in \Phi\left(\mathbf{g}_{\mathbf{c}}, \mathbf{h}_{\mathbf{c}}\right)$, such that

$$
\begin{aligned}
\alpha^{1}(H,-H) & =\alpha^{1}(H, H)=\alpha(H), \\
\alpha^{2}(H,-H) & =-\alpha^{2}(H, H)=\alpha(H), \quad \forall H \in \mathbf{h} .
\end{aligned}
$$

Actually $\mathbf{g}_{\mathbf{c}}^{\alpha^{1}}=\mathbf{C}\left(E_{\alpha}, 0\right)$, and $\mathbf{g}_{\mathbf{c}}^{\alpha^{2}}=\mathbf{C}\left(0, E_{-\alpha}\right)$. This obviously exhausts all the roots of $\left(\mathbf{g}_{\mathbf{c}}, \mathbf{h}_{\mathbf{c}}\right)$. We say the root $\alpha^{i}, i=1,2$ is positive if and only if $\alpha$ is positive. $\Phi\left(\mathbf{g}_{\mathbf{c}}, \mathbf{a}_{\mathbf{c}}\right)$ and $\Phi^{+}\left(\mathbf{g}_{\mathbf{c}}, \mathbf{a}_{\mathbf{c}}\right)$ are those of $\left(\mathbf{g}_{\mathbf{c}}, \mathbf{h}_{\mathbf{c}}\right)$ restricted to $\mathbf{a}_{\mathbf{c}}$, they coincide with $\Phi, \Phi^{+}$, but with multiplicity 2 . Note with this choice of $\Phi^{+}\left(\mathbf{g}_{\mathbf{c}}, \mathbf{a}_{\mathbf{c}}\right), \mathbf{n}_{\mathbf{c}}$ corresponds to negative roots. Let

$$
\rho=\sum_{\alpha \in \Phi^{+}(\mathbf{g}, \mathbf{h})} \alpha ; \quad \rho^{i}=\sum_{\alpha \in \Phi^{+}(\mathbf{g}, \mathbf{h})} \alpha^{i}, i=1,2 ; \quad \rho_{\mathbf{a}}=\sum_{\alpha \in \Phi^{+}\left(\mathbf{g c}, \mathbf{a}_{\mathrm{c}}\right)} \alpha ;
$$


we have natural identification $\rho_{\mathbf{a}}=\rho^{1}+\rho^{2}$, specifically,

$$
\rho_{\mathbf{a}}(H, H)=0, \quad \rho_{\mathbf{a}}(H,-H)=2 \rho(H) .
$$

Lemma (3.4). The bilinear form $\langle$,$\rangle on \mathbf{g}_{\mathbf{c}} \cong \mathbf{g} \oplus \mathbf{g}$ defined by

$$
\left\langle\left(x_{1}, y_{1}\right),\left(x_{2}, y_{2}\right)\right\rangle=\frac{1}{2}\left(B\left(x_{1}, x_{2}\right)-B\left(y_{1}, y_{2}\right)\right)
$$

identifies $\mathbf{a}_{\mathbf{c}} \oplus \mathbf{n}_{\mathbf{c}}$ with $\mathbf{k}_{\mathbf{c}}^{*}$.

Proof. It is straightforward, we simply give the dual bases. Let $\alpha_{j}, j=1,2, \ldots, r k \mathbf{g}$, be the simple roots, $b_{j k}=B\left(H_{\alpha_{j}}, H_{\alpha_{k}}\right), b_{j k}^{-1}$ the matrix element of $\left(b_{j k}\right)^{-1} . H_{j}, E_{ \pm \alpha}$, where $\alpha>0$, is a basis for $\mathbf{g}$. The following are the bases of $\mathbf{k}_{\mathbf{c}}$ and $\mathbf{a}_{\mathbf{c}} \oplus \mathbf{n}_{\mathbf{c}}$, and they are dual to each other:

$$
\begin{array}{cc}
\mathbf{k}_{\mathbf{c}}: & \\
\left(H_{j}, H_{j}\right) & \mathbf{a}_{\mathbf{c}} \oplus \mathbf{n}_{\mathbf{c}}: \\
\left(E_{\alpha}, E_{\alpha}\right) & \left(2 E_{-\alpha}^{-1} H_{k},-b_{j k}^{-1} H_{k}\right), \\
\left(E_{-\alpha}, E_{-\alpha}\right) & \left(0,-2 E_{\alpha}\right)
\end{array}
$$

where $\alpha>0$.

Lemma (3.5). The Lie bracket of $\mathbf{g}^{*} \cong \mathbf{a}_{\mathbf{c}} \oplus \mathbf{n}_{\mathbf{c}},[]:, \wedge^{2} \mathbf{g}^{*} \rightarrow \mathbf{g}^{*}$ induces a map $\delta: \mathbf{g} \rightarrow \wedge^{2} \mathbf{g}$ of the following form:

$$
\delta(x)=\operatorname{ad}_{x}(R), \quad \forall x \in \mathbf{g},
$$

and

$$
R=\frac{1}{2} \sum_{\alpha>0} E_{\alpha} \wedge E_{-\alpha}
$$

The proof is left to the reader. We write down the map $\delta$ here:

$$
\begin{aligned}
\delta\left(H_{j}\right) & =0 \\
\delta\left(E_{\alpha}\right) & =\frac{1}{2} E_{\alpha} \wedge H_{\alpha}-\frac{1}{2} \sum_{\beta, \gamma>0, \beta+\gamma=\alpha} N_{\beta, \gamma} E_{\beta} \wedge E_{\gamma}, \\
\delta\left(E_{-\alpha}\right) & =\frac{1}{2} E_{-\alpha} \wedge H_{\alpha}-\frac{1}{2} \sum_{\beta, \gamma>0, \beta+\gamma=\alpha} N_{\beta, \gamma} E_{-\beta} \wedge E_{-\gamma},
\end{aligned}
$$

where $\alpha>0$.

Lemma (3.6). For any $x \in \mathbf{g} \cong \mathbf{k}_{\mathbf{c}} \subset \mathbf{g}_{\mathbf{c}}, y \in \mathbf{g}^{*} \cong \mathbf{a}_{\mathbf{c}} \oplus \mathbf{n}_{\mathbf{c}} \subset \mathbf{g}_{\mathbf{c}}$,

$$
[x, y]:=-\left(\operatorname{ad}_{x}^{*}(y)-\operatorname{ad}_{y}^{*}(x)\right)
$$

coincides with the Lie bracket of $\mathbf{g}_{\mathbf{c}}=\mathbf{k}_{\mathbf{c}} \oplus \mathbf{a}_{\mathbf{c}} \oplus \mathbf{n}_{\mathbf{c}}$.

\section{Proposition (3.7).}

1) $\left(\mathbf{k}_{\mathbf{c}}, \mathbf{a}_{\mathbf{c}} \oplus \mathbf{n}_{\mathbf{c}}, \mathbf{g}_{\mathbf{c}}\right)$ is a Manin triple;

2) $(G, \pi), \pi(g)=l_{g^{*}} R-r_{g^{*}} R$, for $g \in G$, is a Poisson Lie group.

Definition (3.8). A Poisson Hopf algebra is a commutative Hopf algebra with a compatible Poisson bracket, i.e., $\{a, b c\}=b\{a, c\}+\{a, b\} c$. We also have the dual object, co-Poisson Hopf algebra. 
Theorem (3.9). [11]

1) The Lie bialgebra structure $(\mathbf{g}, \delta)$ induces a unique co-Poisson Hopf algebra structure on $U(\mathbf{g})$;

2) The Poisson Lie group structure $(\mathbf{G}, \pi)$ induces a unique Poisson Hopf algebra structure on $\mathbf{C}[G]$, and on $\mathbf{C}^{f}(G) ; \mathbf{C}^{f}(G)$ is dual to $U(\mathbf{g})$.

This gives the quasiclassical limit of our quantum groups.

Definition (3.10). A quantization of Poisson Hopf algebra $A_{0}$ over $\mathbf{C}$ is a Hopf algebra $A$ over $\mathbf{C}[[h]]$ such that

(a) $A / h A=A_{0}$ as Hopf algebra over $\mathbf{C}$,

(b) $A$ is a free $\mathbf{C}[[h]]$-module,

(c) $\forall a, b \in A,(a b-b a) / h \bmod (h)=\{a \bmod (h), b \bmod (h)\}$, where $\{$,$\} is the$ Poisson bracket of $A_{0}$.

The notion of quantization of co-Poisson Hopf algebra is similar.

Theorem (3.11). [11] The co-Poisson Hopf algebra $(U(\mathbf{g}), \delta)$ has a unique quantization $U_{h}(\mathbf{g})$.

This is the quantized universal enveloping algebra of Drinfeld and Jimbo $[11,21]$.

Corollary (3.12). The Poisson Hopf algebra $\left(\mathbf{C}^{f}(G), \pi\right)$ has a unique quantization $A_{h}^{f}(G)$.

Theorem (3.13). The Poisson Hopf algebra $(\mathbf{C}[G], \pi)$ has a quantization $A_{h}[G]$.

Definition (3.14). Let $\mathbf{C}_{h}^{f}(G)$ be the h-adic completion of $A_{h}^{f}(G) \otimes_{\mathrm{C}_{[[h]]}} \mathbf{C}((h))$ and $\mathbf{C}_{h}[G]$ the h-adic completion of $A_{h}[G] \otimes_{\mathbf{C}_{[[h]]}} \mathbf{C}((h))$.

See $[31,29,35]$ for more discussion of $\mathbf{C}_{h}[G]$. It is called quantum coordinate algebra there. For the purpose of this paper we do not need an explicit construction of $\mathbf{C}_{h}[G]$.

Example (3.15). $G=S L(2, \mathbf{C}), \mathbf{g}=\operatorname{sl}(2, \mathbf{C})$.

$U_{h}(\mathbf{g})$ is a Hopf algebra generated by $H, E^{+}, E^{-}$via

$$
\begin{aligned}
{\left[H, E_{ \pm}\right] } & = \pm E_{ \pm} \\
{\left[E_{+}, E_{-}\right] } & =\frac{e^{h H}-e^{-h H}}{e^{h}-e^{-h}} \\
\Delta(H) & =1 \otimes H+H \otimes 1 \\
\Delta\left(E_{ \pm}\right) & =E_{ \pm} \otimes \exp \left(\frac{1}{2} h H\right)+\exp \left(-\frac{1}{2} h H\right) \otimes E_{ \pm}, \\
\varepsilon(H) & =0, \quad \varepsilon\left(E_{ \pm}\right)=0, \\
S(H) & =-H, \quad S\left(E_{ \pm}\right)=-e^{ \pm h} E_{ \pm} .
\end{aligned}
$$

$\mathbf{C}_{h}[G]$ is a Hopf algebra generated by $a, b, c, d$ via

$$
a b=e^{-h} b a, \quad a c=e^{-h} c a, \quad d b=e^{h} b d, \quad d c=e^{h} c d,
$$




$$
\begin{aligned}
& b c=c b, \quad a d-d a=\left(e^{-h}-e^{h}\right) b c, \quad a d-e^{-h} b c=1, \\
& \Delta\left(\begin{array}{ll}
a & b \\
c & d
\end{array}\right)=\left(\begin{array}{ll}
a & b \\
c & d
\end{array}\right) \hat{\otimes}\left(\begin{array}{ll}
a & b \\
c & d
\end{array}\right),
\end{aligned}
$$

where ${ }^{\wedge}$ indicates matrix multiplication,

$$
\begin{aligned}
& \varepsilon(a)=\varepsilon(d)=1, \quad \varepsilon(b)=\varepsilon(c)=0, \\
& S(a)=d, \quad S(d)=a, \quad S(b)=-e^{h} b, \quad S(c)=-e^{-h} c .
\end{aligned}
$$

\section{A Spectral Sequence}

We start the computation of the Hochschild and cyclic homology of quantum groups in this section. Our major technical tool is the spectral sequence.

First a remark about notation. Note we have natural identification $K_{\mathbf{C}} \cong G$. In this and the next section we will use $K_{C}$ to denote the complex Lie group $G$ and reserve $G$ for the real Lie group structure of $G$. All Lie algebras are complex Lie algebras and we will use the identification $\mathbf{g} \cong \mathbf{k}_{\mathbf{c}}, \mathbf{g}^{*} \cong \mathbf{a}_{\mathbf{c}} \oplus \mathbf{n}_{\mathbf{c}}$ without further notice.

We will denote quantum group $\mathbf{C}_{h}^{f}\left(K_{\mathbf{C}}\right)$, or $\mathbf{C}_{h}\left[K_{\mathbf{C}}\right]$ by $A$ and the corresponding undeformed Hopf algebra by $A_{0}$. The $h$ (Planck constant) introduces a natural filtration in the Hochschild complex. The associated spectral sequence has $E_{*}^{\infty}=H H_{*}(A)$. In this section we identify $E_{*}^{2}$ as Lie algebra homology $H_{*}^{*}\left(\mathbf{g}^{*}, A_{0}\right)$.

Note as vector space over $\mathbf{C}, A=\mathbf{C}((h)) \otimes_{\mathbf{C}} A_{0}$, therefore

$$
\mathrm{C}_{n}(A)=A \otimes_{\left.\mathrm{C}_{(}(h)\right)} A \otimes_{\mathrm{C}((h))} \cdots \otimes_{\mathrm{C}(h))} A \cong \mathbf{C}((h)) \otimes_{\mathrm{C}} C_{n}\left(A_{0}\right)
$$

as $\mathbf{C}$ vector space. The Planck constant introduces the filtration

$$
C_{*}(A) \supset h C_{*}(A) \supset h^{2} C_{*}(A) \supset \cdots,
$$

which obviously makes (C. $(A), d)$ into a filtered complex. Intuitively we can write $d=d_{0}+h d_{1}+h^{2} d_{2}+\cdots$ and the filtered complex as

$$
\begin{gathered}
C_{0}\left(A_{0}\right) \stackrel{d_{0}}{\longleftarrow} C_{1}\left(A_{0}\right) \stackrel{d_{0}}{\longleftarrow} C_{2}\left(A_{0}\right) \stackrel{d_{0}}{\longleftarrow} \cdots \\
h C_{0}\left(A_{0}\right) \stackrel{d_{0}}{\longleftarrow} h C_{1}\left(A_{0}\right) \stackrel{d_{d_{1}}}{\longleftarrow} h C_{2}\left(A_{0}\right) \stackrel{d_{0}}{\longleftarrow} \cdots \cdot \\
\vdots \\
h d_{1}
\end{gathered}
$$

Actually $d_{n}$ is well-defined only when $d_{0}+h d_{1}+\cdots+h^{n-1} d_{n-1}=0$. That is exactly the situation when we use $d_{1}, d_{2}$ as it is in general for spectral sequence. 
It is quite obvious that $d_{0}$ reduces all the computation to that of $A_{0}$, thus $E_{*}^{1}=H_{*}\left(C \cdot(A), d_{0}\right)$ is simply the Hochschild homology of $A_{0}$. From HochschildKostant-Rosenberg theorem we have

Lemma (4.1).

$$
E_{n}^{1}=H H_{n}\left(A_{0}\right)=\Omega^{n}\left(K_{\mathrm{C}}\right)
$$

where the differential forms take algebraic or formal coefficients.

The main result of this section is the following theorem.

Theorem (4.2).

$$
E_{n}^{2}=H_{n}\left(\mathbf{g}^{*}, A_{0}\right)
$$

i.e., the $E^{2}$ term is the Lie algebra homology of $\mathbf{g}^{*} \cong \mathbf{a}_{\mathbf{c}} \oplus \mathbf{n}_{\mathbf{c}}$ with coefficient in $A_{0}$. For any $X \in \mathrm{g}^{*}$, the action of $X$ on $A_{0}$ is

$$
\sigma(X)=\sigma_{0}(X)+\rho_{\mathbf{a}}(X),
$$

where $\rho_{\mathrm{a}}$ is the half sum of the positive restricted roots and $\sigma_{0}(X)$ coincides with the infinitesimal left translation of $X \in \mathbf{g}^{*} \subset \mathbf{g}_{\mathbf{c}}$ on

$$
A_{0} \cong\left\{f \in \mathbf{C}^{?}\left(G_{\mathbf{C}}\right) \mid f\left(g e^{X}\right)=e^{\rho_{\mathbf{a}}(X)} f(g), \forall g \in G_{\mathbf{C}}, X \in \mathbf{g}^{*}\right\} .
$$

Here $\mathbf{C}^{?}\left(G_{\mathbf{C}}\right)$ denotes either $\mathbf{C}^{f}\left(G_{\mathbf{C}}\right)$ ( formal functions) or $\mathbf{C}^{a}\left(G_{\mathbf{C}}\right)$ (rational functions on $G_{\mathbf{C}}$ which are regular on $K_{\mathbf{C}} A_{\mathbf{C}} N_{\mathbf{C}}$ ) depending on whether $A_{0}$ is formal or algebraic functions on $K_{\mathrm{C}}$.

The rest of the section is for the proof of this theorem. We will identify the complex $\left(E .{ }^{1}, d_{1}\right)$ as the complex for the Lie algebra homology $H_{*}\left(\mathrm{~g}^{*}, A_{0}\right)$, and then give the geometrical meaning of the $\mathrm{g}^{*}$ action on $A_{0}$. Let us introduce a few notations. $I_{a}, a=1,2, \ldots, \operatorname{dim} \mathbf{g}$, is a basis for $\mathbf{g}$; $J^{a}$ the dual basis; $X_{a}^{l}\left(X_{a}^{r}\right)$ the left (right) invariant vector fields on $K_{\mathbf{C}}$ such that $X_{a}^{l}(e)=X_{a}^{r}(e)=I_{a} ; \omega_{l}^{a}\left(\omega_{r}^{a}\right)$ the left (right) invariant 1 -forms on $K_{\mathrm{C}}$ such that $\omega_{l}^{a}(e)=\omega_{r}^{a}(e)=J^{a} . \Delta, S, \varepsilon$ denote the coproduct, antipode, and counit of $A ; \Delta_{0}, S_{0}, \varepsilon_{0}$ those of $A_{0} ; x * y$ the product in $A$ and $x y$ that of $A_{0}$. We identify $A \cong \mathbf{C}((h)) \otimes_{\mathbf{C}} A_{0}$ as vector space over $\mathbf{C}$.

Lemma (4.3). $\forall f_{0}, f_{1} \in A_{0}, d_{1}: E_{1}^{1} \rightarrow E_{0}^{1}$ has the form

$$
d_{1}\left(f_{0} \otimes f_{1}\right)=-\left(X_{a}^{r} f_{1}\right)(e)\left(Y^{a}-f^{a}\right) f_{0},
$$

where

$$
\begin{aligned}
Y^{a} f_{0} & =\left\langle\pi, \omega_{r}^{a} \wedge d f_{0}\right\rangle, \\
f^{a} & =\left\langle\pi, d \omega_{r}^{a}\right\rangle .
\end{aligned}
$$

Proof. Let $\Delta\left(f_{1}\right)=\sum_{j} A_{j} \otimes B_{j}$, since $\varepsilon\left(f_{1}\right)=\sum_{j} A_{j} \otimes B_{j}^{s}$ we have

$$
\begin{aligned}
d\left(f_{0} \otimes f_{1}\right) & =\rho\left(f_{1}\right) f_{0}-\varepsilon\left(f_{1}\right) f_{0}=\sum_{j} B_{j}^{s} * f_{0} * A_{j}-\sum_{j} A_{j} * B_{j}^{s} * f_{0} \\
& =\sum_{j} B_{j}^{s} *\left(f_{0} * A_{j}-A_{j} * f_{0}\right)-\sum_{j}\left(A_{j} * B_{j}^{s}-B_{j}^{s} * A_{j}\right) * f_{0} \\
& =h \sum_{j} b_{j}^{s_{0}}\left\{f_{0}, a_{j}\right\}-h \sum_{j}\left\{a_{j}, b_{j}^{s_{0}}\right\} f_{0}+o\left(h^{2}\right),
\end{aligned}
$$


where $\Delta_{0}\left(f_{1}\right)=\sum_{j} a_{j} \otimes b_{j} . d_{1}$ is the $h$ linear term of this. Thus we get

$$
d_{1}\left(f_{0} \otimes f_{1}\right)=-\sum_{j}\left\{a_{j}, f_{0}\right\} b_{j}^{s_{0}}-f_{0} \sum_{j}\left\{a_{j}, b_{j}^{s_{0}}\right\} .
$$

Note on Lie group we have $d f=\omega_{l}^{a}\left(X_{a}^{l} f\right)=\omega_{r}^{a}\left(X_{a}^{r} f\right)$. Therefore

$$
\begin{aligned}
\sum_{j} b_{j}^{s_{0}}\left\{a_{j}, f_{0}\right\} & =\sum_{j}\left\langle\pi, \omega_{r}^{a} \wedge d f_{0}\right\rangle\left(X_{a}^{r} a_{j}\right) b_{j}^{s_{0}}=\left(Y^{a} f_{0}\right) \sum_{j}\left(X_{j}^{r} a_{j}\right) b_{j}^{s_{0}}(g) \\
& =\left(Y^{a} f_{0}\right) \sum_{j} \lim _{t \rightarrow 0} \frac{1}{t}\left(a_{j}\left(e^{t I_{a}} g\right)-a_{j}(g)\right) b_{j}\left(g^{-1}\right) \\
& =\left(Y^{a} f_{0}\right) \lim _{t \rightarrow 0} \frac{1}{t}\left(f_{1}\left(e^{t I_{a}}\right)-f_{1}(e)\right)=\left(X_{a}^{r} f_{1}\right)(e)\left(Y^{a} f_{0}\right)
\end{aligned}
$$

where we have used $b_{j}^{s_{0}}(g)=b_{j}\left(g^{-1}\right)$, and $\sum_{j} a_{j}(g) b_{j}(h)=f_{1}(g h)$. On the other hand,

$$
\sum_{j}\left\{a_{j}, b_{j}^{s_{0}}\right\}=\left\langle\pi, \omega_{r}^{a} \wedge \omega_{r}^{b}\right\rangle \sum_{j}\left(X_{a}^{r} a_{j}\right)\left(X_{b}^{r} b_{j}^{s_{0}}\right)
$$

where

$$
\begin{aligned}
\sum_{j}\left(X_{a}^{r} a_{j}\right)\left(X_{b}^{r} b_{j}^{s_{0}}\right) & =\sum_{j} \lim _{t, s \rightarrow 0} \frac{1}{t s}\left(a_{j}\left(e^{t I_{a}} g\right)-a_{j}(g)\right)\left(b_{j}\left(g^{-1} e^{-s I_{b}}\right)-b_{j}\left(g^{-1}\right)\right) \\
& =\sum_{j} \lim _{t, s \rightarrow 0} \frac{1}{t s}\left(f_{1}\left(e^{t I_{a}} e^{-s I_{b}}\right)-f_{1}\left(e^{t I_{a}}\right)-f_{1}\left(e^{-s I_{b}}\right)+f_{1}(e)\right) \\
& =\left(X_{a}^{r} X_{b}^{r} f_{1}\right)(e) .
\end{aligned}
$$

Thus

$$
\begin{aligned}
\sum_{j}\left\{a_{j}, b_{j}^{s_{0}}\right\} & =\left\langle\pi, \omega_{r}^{a} \wedge \omega_{r}^{b}\right\rangle\left(X_{a}^{r} X_{b}^{r} f_{1}\right)(e)=\frac{1}{2}\left\langle\pi, \omega_{r}^{a} \wedge \omega_{r}^{b}\right\rangle\left(X_{a}^{r} X_{b}^{r}-X_{b}^{r} X_{a}^{r}\right) f_{1}(e) \\
& =-\frac{1}{2} f_{a b}^{c}\left\langle\pi, \omega_{r}^{a} \wedge \omega_{r}^{b}\right\rangle\left(X_{c}^{r} f_{1}\right)(e)=-\left\langle\pi, d \omega_{r}^{c}\right\rangle\left(X_{c}^{r} f_{1}\right)(e),
\end{aligned}
$$

where $\left[I_{a}, I_{b}\right]=f_{a b}^{c} I_{c}$, and we have used $\left[X_{a}^{r}, X_{b}^{r}\right]=-f_{a b}^{c} X_{c}^{r}, d \omega_{r}^{c}=\frac{1}{2} f_{a b}^{c} \omega_{r}^{a} \wedge \omega_{r}^{b}$.

\section{Lemma (4.4).}

$$
\left[\sigma\left(J^{a}\right), \sigma\left(J^{b}\right)\right]=\sigma\left(\left[J^{a}, J^{b}\right]\right),
$$

where $\sigma\left(J^{a}\right)=Y^{a}-f^{a}$, so $\sigma$ defines a representation of $\mathbf{g}^{*}$ on $A_{0}$.

Proof. It is simple calculation. We remark that $\left[Y^{a}, Y^{b}\right]=\lambda_{c}^{a b} Y^{c}$, where $\lambda_{c}^{a b}$ is the structure constant of $\mathbf{g}^{*}$, is equivalent to the requirement that the Poisson bracket defined by $\left\{f_{1}, f_{2}\right\}=\left\langle\pi, d f_{1} \wedge d f_{2}\right\rangle$ satisfies Jacobi identity.

Consider the Lie algebra homology of $\mathbf{g}^{*}$ with coefficients in $A_{0}, H_{*}\left(\mathbf{g}^{*}, A_{0}\right)$, where $\mathbf{g}^{*}$ acts on $A_{0}$ via $\sigma$. It can be computed by the standard complex

$$
0 \longleftarrow A_{0} \stackrel{d_{\mathbf{g}^{*}}}{\longleftarrow} A_{0} \otimes \mathbf{g}^{*} \stackrel{d^{\mathbf{g}^{*}}}{\longleftarrow} A_{0} \otimes \wedge^{2} \mathbf{g}^{*} \stackrel{d^{*}}{\longleftarrow} \cdots,
$$


where $d_{\mathbf{g}^{*}}: A_{0} \otimes \wedge^{n} \mathbf{g}^{*} \rightarrow A_{0} \otimes \wedge^{n-1} \mathbf{g}^{*}$ is such that

$$
\begin{aligned}
d_{\mathbf{g}^{*}}\left(f \otimes y_{1} \wedge y_{2} \wedge \cdots \wedge y_{n}\right) \\
=\sum_{i=1}^{n}(-1)^{i-1} \sigma\left(y_{i}\right) f \otimes y_{1} \wedge \cdots \wedge \hat{y}_{i} \cdots \wedge y_{n} \\
\quad+\sum_{i<j}(-1)^{i+j-1} f \otimes\left[y_{i}, y_{j}\right] y_{1} \wedge \cdots \wedge \hat{y}_{i} \cdots \wedge \hat{y}_{j} \cdots \wedge y_{n} .
\end{aligned}
$$

And we have seen that $E_{*}^{2}=H_{*}\left(E_{\bullet}^{1}, d_{1}\right)$, with $E_{*}^{1}=H H_{n}\left(A_{0}\right) \cong \Omega^{n}\left(A_{0}\right) \cong A_{0} \otimes \wedge^{n} \mathbf{g}^{*}$.

Define a map $\eta: H H_{n}^{*}\left(A_{0}\right) \rightarrow A_{0} \otimes \wedge^{n} \mathbf{g}^{*}$ by

$$
\begin{aligned}
& \eta\left(f_{0} \otimes \sum_{s \in S_{n}}(-1)^{l(s)} f_{s(1)} \otimes f_{s(2)} \otimes \cdots \otimes f_{s(n)}\right) \\
& \quad=(-1)^{n} f_{0} \otimes\left(\left(d f_{1}\right)(e) \wedge\left(d f_{2}\right)(e) \wedge \cdots \wedge\left(d f_{n}\right)(e)\right),
\end{aligned}
$$

where $S_{n}$ is the permutation group, $l(s)$ is the length of the permutation, $e$ is the identity of the group $K_{\mathrm{C}}$.

\section{Lemma (4.5).}

1) The following diagram is commutative

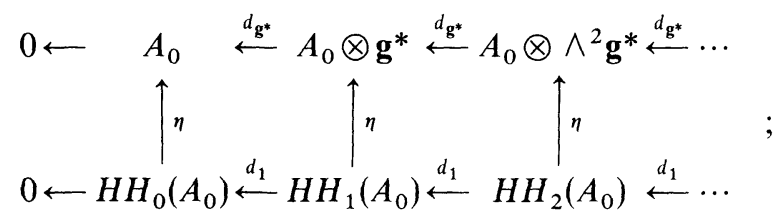

2) $\eta$ introduces an isomorphism of complexes. Proof. 1) Suffice it to check for $n=2$. We will use $\left.\frac{\partial}{\partial h}\right|_{h=0} d$ for $d_{1}$ since $d_{1}$ is the
$h$ linear part of $d$.

$$
\begin{aligned}
\eta \cdot d_{1}\left(f_{0} \otimes f_{1} \otimes f_{2}-f_{0} \otimes f_{2} \otimes f_{1}\right) \\
=\left.\eta \cdot \frac{\partial}{\partial h}\right|_{h=0} d\left(f_{0} \otimes f_{1} \otimes f_{2}-f_{0} \otimes f_{2} \otimes f_{1}\right) \\
=\left.\eta \cdot \frac{\partial}{\partial h}\right|_{h=0}\left(\rho\left(f_{1}\right) f_{0} \otimes f_{2}-f_{0} \otimes f_{1} * f_{2}+\varepsilon\left(f_{2}\right) f_{0} \otimes f_{1}\right. \\
\left.\quad-\rho\left(f_{2}\right) f_{0} \otimes f_{1}+f_{0} \otimes f_{2} * f_{1}-\varepsilon\left(f_{1}\right) f_{0} \otimes f_{2}\right) \\
=\eta\left(d_{1}\left(f_{0} \otimes f_{1}\right) \otimes f_{2}-d_{1}\left(f_{0} \otimes f_{2}\right) \otimes f_{1}-f_{0} \otimes\left\{f_{1}, f_{2}\right\}\right) \\
=\sigma\left(\left(d f_{1}\right)(e)\right) f_{0} \otimes\left(d f_{2}\right)(e)-\sigma\left(\left(d f_{2}\right)(e)\right) f_{0} \otimes\left(d f_{1}\right)(e)+f_{0} \otimes\left(d\left\{f_{1}, f_{2}\right\}\right)(e) .
\end{aligned}
$$

This will equal $d_{\mathrm{g}^{*}} \cdot \eta\left(f_{0} \otimes f_{1} \otimes f_{2}-f_{0} \otimes f_{2} \otimes f_{1}\right)$, provided

Actually,

$$
\left(d\left\{f_{1}, f_{2}\right\}\right)(e)=\left[\left(d f_{1}\right)(e),\left(d f_{2}\right)(e)\right] \text {. }
$$

$$
\begin{aligned}
\left(d\left\{f_{1}, f_{2}\right\}\right)(e) & =\omega_{l}^{a} X_{a}^{l}\left\langle\pi, d f_{1} \wedge d f_{2}\right\rangle(e) \\
& =\omega_{l}^{a}\left\langle\mathscr{L}_{X_{a}^{l}} \pi, d f_{1} \wedge d f_{2}\right\rangle(e)+\omega_{l}^{a}\left\langle\pi, \mathscr{L}_{X_{a}^{l}}\left(d f_{1} \wedge d f_{2}\right)\right\rangle(e) .
\end{aligned}
$$


The second term vanishes since $\pi(e)=0$, and $\left(\mathscr{L}_{X_{a}^{l}} \pi\right)(e)=\operatorname{ad}_{I_{a}}(R)=\delta\left(I_{a}\right)$, thus

$$
\begin{aligned}
\left(d\left\{f_{1}, f_{2}\right\}\right)(e) & =J^{a}\left\langle\delta\left(I_{a}\right),\left(d f_{1}\right)(e) \wedge\left(d f_{2}\right)(e)\right\rangle=J^{a}\left\langle I_{a},\left[\left(d f_{1}\right)(e),\left(d f_{2}\right)(e)\right]\right\rangle \\
& =\left[\left(d f_{1}\right)(e),\left(d f_{2}\right)(e)\right] .
\end{aligned}
$$

2) Actually $\eta=\Gamma \cdot \xi$, where $\xi$ is the isomorphism between the new and the standard complexes for the Hochschild homology of Hopf algebras as defined in (2.18), and $\Gamma$ is the Hochschild-Kostant-Rosenberg isomorphism $\Gamma: H H_{n}\left(A_{0}\right) \stackrel{\sim}{\rightarrow} \Omega^{n}\left(A_{0}\right)$ in Lemma (2.1), given explicitly by

$$
\Gamma\left(\sum_{s \in S_{n}}(-1)^{l(s)} f_{0} \otimes f_{s(1)} \otimes f_{s(2)} \otimes \cdots \otimes f_{s(n)}\right)=f_{0} d f_{1} \wedge d f_{2} \wedge \cdots \wedge d f_{n},
$$

$S_{n}$ is the permutation group and $l(s)$ is the length of the permutation.

We therefore have proven the first part of the main theorem.

\section{Proposition (4.6).}

$$
E_{n}^{2}=H_{n}\left(\mathbf{g}^{*}, A_{0}\right), \quad \text { where } \mathbf{g}^{*} \text { acts on } A_{0} \text { by } \sigma \text { as in Lemma (4.4). }
$$

Remark. In [3] Brylinsky defines a complex associated with any Poisson manifold. We remark that the complex $\left(E ., d_{1}\right)$, which we have identified with the standard complex of the Lie algebra homology $H_{*}\left(\mathbf{g}^{*}, A_{0}\right)$, is isomorphic to the Brylinsky complex associated with the Poisson Lie group $\left(K_{\mathbf{C}}, \pi\right)$. This is most evident if we use the standard complex for the Hochschild homology.

We will now give the geometrical meaning of the action $\sigma$ of $\mathbf{g}^{*}$ on $A_{0}$. Recall that our Lie bialgebra $\left(\mathbf{g}, \mathbf{g}^{*}, \mathbf{g} \bowtie \mathbf{g}^{*}\right)$ is $\left(\mathbf{k}_{\mathbf{c}}, \mathbf{a}_{\mathbf{c}} \oplus \mathbf{n}_{\mathbf{c}}, \mathbf{g}_{\mathbf{c}}\right)$, and the corresponding local double Lie group [28] $\left(G, G^{*}, G \bowtie G^{*}\right)$ is $\left(K_{\mathbf{C}}, A_{\mathbf{C}} N_{\mathbf{C}}, G_{\mathbf{C}}\right)$. We observe that $Y^{a}$ is the right dressing action $[28,39]$ of the Poisson Lie group $(G, \pi)$. By a result of Lu and Weinstein, we have the following

Lemma (4.7) [28]. $Y^{a}$ coincides with the infinitesimal left translation of $J^{a} \in \mathbf{g}^{*} \subset \mathbf{g}_{\mathbf{c}}$ on the open $K_{\mathbf{C}}$ orbit of the $G_{\mathbf{C}}$ homogeneous space $G_{\mathbf{C}} / A_{\mathbf{C}} N_{\mathbf{C}}$. Therefore the $Y^{a}$ action on $A_{0}$ coincides with the left translation by $\mathbf{g}^{*} \subset \mathbf{g}_{\mathbf{C}}$ on

$$
A_{0} \cong\left\{f \in \mathbf{C}^{?}\left(G_{\mathbf{C}}\right) \mid f(g h)=f(g), \forall g \in G_{\mathbf{C}}, h \in G^{*}\right\},
$$

where $\mathbf{C}^{?}\left(G_{\mathbf{C}}\right)$ denotes either $\mathbf{C}^{a}\left(G_{\mathbf{C}}\right)$ or $\mathbf{C}^{f}\left(G_{\mathbf{C}}\right)$ depending on whether $A_{0}$ is algebraic or formal functions on $K_{\mathbf{C}}$.

Lemma (4.8). The zeroth order term of $\sigma$ can be written as

$$
f^{a}:=\left\langle\pi, d \omega_{r}^{a}\right\rangle=2\left\langle H_{\rho}^{l}-H_{\rho}^{r}, \omega_{r}^{a}\right\rangle,
$$

where $H_{\rho}^{l}, H_{\rho}^{r}$ are respectively left, right invariant vector fields on $K_{\mathrm{C}}$ such that they coincide at the identity with

$$
H_{\rho}=\frac{1}{2} \sum_{\alpha>0} H_{\alpha} .
$$

Proof. Recall $R=\sum_{\alpha>0} E_{\alpha} \wedge E_{-\alpha}$, therefore

$$
\pi=\sum_{\alpha>0} X_{\alpha}^{l} \wedge X_{-\alpha}^{l}-\sum_{\alpha>0} X_{\alpha}^{r} \wedge X_{-\alpha}^{r}
$$


Use

$$
\langle X \wedge Y, d \omega\rangle=X\langle Y, \omega\rangle-Y\langle X, \omega\rangle-\langle[X, Y], \omega\rangle,
$$

we have

$$
\begin{aligned}
\sum_{\alpha>0}\left\langle X_{\alpha}^{l} \wedge X_{-\alpha}^{l}, d \omega_{r}^{a}\right\rangle & =\sum_{\alpha>0}\left\langle H_{\alpha}^{l}, \omega_{r}^{a}\right\rangle, \\
\sum_{\alpha>0}\left\langle X_{\alpha}^{r} \wedge X_{-\alpha}^{r}, d \omega_{r}^{a}\right\rangle & =\sum_{\alpha>0}\left\langle H_{\rho}^{r}, \omega_{r}^{a}\right\rangle .
\end{aligned}
$$

\section{Lemma (4.9).}

1) $\forall Y \in \mathbf{g}^{*} \cong \mathbf{a}_{\mathbf{c}} \oplus \mathbf{n}_{\mathbf{c}}$,

$$
2\left\langle H_{\rho}, Y\right\rangle=\rho_{\mathrm{a}}(Y) ;
$$

2) $\forall X \in \mathbf{g} \subset \mathbf{g}_{\mathbf{C}}$, and $\omega_{r}(e)=Y \in \mathbf{g}^{*} \subset \mathbf{g}_{\mathbf{C}}$,

$$
2\left\langle H_{\rho}^{l}, \omega_{r}\right\rangle\left(e^{t X}\right)=\rho_{\mathrm{a}}\left(e^{-t X} Y e^{t X}\right),
$$

where $\rho_{\mathbf{a}}$ is thought of as a linear form on $\mathbf{g}_{\mathbf{c}}=\mathbf{k}_{\mathbf{c}} \oplus \mathbf{a}_{\mathbf{c}} \oplus \mathbf{n}_{\mathbf{c}}$ which only takes non-zero value on $\mathbf{a}_{\mathrm{c}}$.

Proof. 1) Since both sides are zero when $Y$ is in $\mathbf{n}_{\mathbf{c}}$, we only need to check for $\mathbf{a}_{\mathbf{c}}$. With $\mathbf{g} \cong \mathbf{k}_{\mathbf{c}}$ we have $H_{\rho}=\left(H_{\rho}, H_{\rho}\right)$. Thus $\forall(H,-H) \in \mathbf{a}_{\mathbf{c}}$, we get

$$
\begin{aligned}
2\left\langle\left(H_{\rho}, H_{\rho}\right),(H,-H)\right\rangle & =2 \frac{1}{2}\left(B\left(H_{\rho}, H\right)-B\left(H_{\rho},-H\right)\right) \\
& =2 \rho(H)=\rho_{\mathbf{a}}((H,-H)) .
\end{aligned}
$$

2)

$$
\begin{aligned}
2\left\langle H_{\rho}^{l}, \omega_{r}\right\rangle\left(e^{t X}\right) & =2\left\langle\operatorname{Ad}_{e^{t X}}\left(H_{\rho}\right), Y\right\rangle \\
& =2\left\langle H_{\rho}, \operatorname{Ad}_{e^{t X}}^{*}(Y)\right\rangle=\rho_{\mathrm{a}}\left(\operatorname{Ad}_{e^{t X}}^{*}(Y)\right) .
\end{aligned}
$$

Now observe that if we consider $X, Y$ as in $\mathbf{g} \bowtie \mathbf{g}^{*}$ then the $\mathbf{g}^{*}$ part of $e^{-t X} Y e^{t X}$ coincides with $\operatorname{Ad}_{e^{t X}}^{*}(Y)$. Actually,

$$
\operatorname{ad}_{X}(Y):=[X, Y]=-\operatorname{ad}_{X}^{*}(Y)+\operatorname{ad}_{Y}^{*}(X),
$$

with $\operatorname{ad}_{X}^{*}(Y) \in \mathbf{g}^{*}, \operatorname{ad}_{Y}^{*}(X) \in \mathbf{g}$ and $\mathbf{g}$ is a subalgebra, thus the $\mathbf{g}^{*}$ part of $\operatorname{ad}_{X} \operatorname{ad}_{X}(Y)$ is from $\operatorname{ad}_{X}\left(-\operatorname{ad}_{X}^{*}(Y)\right)$ which in turn is $\operatorname{ad}_{X}^{*} \operatorname{ad}_{X}^{*}(Y)$. Continue this argument, we see that the $\mathrm{g}^{*}$ part of $\left(\operatorname{ad}_{X}\right)^{n}(Y)$ is $(-1)^{n}\left(\operatorname{ad}_{X}^{*}\right)^{n}(Y)$. Therefore the $\mathrm{g}^{*}$ part of

is

$$
\operatorname{Ad}_{e^{-t X}}(Y)=\sum_{n=0}^{\infty}(-1)^{n} \frac{t^{n}}{n !}\left(\operatorname{ad}_{X}\right)^{n}(Y)
$$

$$
\sum_{n=0}^{\infty} \frac{t^{n}}{n !}\left(\operatorname{ad}_{X}^{*}\right)^{n}(Y)=\operatorname{Ad}_{e^{t X}}^{*}(Y) .
$$

Since $\rho_{\mathbf{a}}$ is zero on $\mathbf{g}$, we have

$$
\rho_{\mathrm{a}}\left(\operatorname{Ad}_{e^{t X}}^{*}(Y)\right)=\rho_{\mathrm{a}}\left(\operatorname{Ad}_{e^{-t X}}(Y)\right) .
$$

Let us write the action of $\mathbf{g}^{*}$ as

$$
\sigma=\sigma_{0}+\rho_{\mathbf{a}},
$$


where

$$
\sigma_{0}\left(J^{a}\right)=Y^{a}-2\left\langle H_{\rho}^{l}, \omega_{r}^{a}\right\rangle .
$$

As a result of Lemma (4.7) and Lemma (4.9), we have the following

Proposition (4.10). The $\sigma_{0}$ action on $A_{0}$ coincides with the left translation by $\mathbf{g}^{*} \cong \mathbf{a}_{\mathbf{c}} \oplus \mathbf{n}_{\mathbf{c}} \in \mathbf{g}_{\mathbf{c}}$ on

$$
A_{0} \cong\left\{f \in \mathbf{C}^{?}\left(G_{\mathbf{C}}\right) \mid f\left(g e^{X}\right)=e^{\rho_{\mathbf{a}}(X)} f(g), \forall g \in G_{\mathbf{C}}, X \in \mathbf{g}^{*}\right\},
$$

where $\mathbf{C}^{?}\left(G_{\mathbf{C}}\right)$ denotes either $\mathbf{C}^{a}\left(G_{\mathbf{C}}\right)$ or $\mathbf{C}^{f}\left(G_{\mathbf{C}}\right)$.

Proof. Note the $\sigma_{0}$ is a differential operator on $G$ with holomorphic coefficients, it is enough to consider its action on the formal power series at the identity. Lemma (4.7) ensures the vector field part. For the zero order part, on the one hand, Lemma (4.9) gives $\rho_{\mathrm{a}}\left(-\operatorname{Ad}_{e^{-t X}}(Y)\right)$ for a point $g=e^{t X}$ in the neighborhood of identity. On the other hand, the left translation of $Y$ acts as $(-Y) g=g\left(-g^{-1} Y g\right)$, the $\mathbf{g}^{*}$ part contributes a constant, which according to (4.21) is $\rho_{\mathbf{a}}\left(-\operatorname{Ad}_{e^{-t X}}(Y)\right)$. The two actions agree to all powers of $t$.

This is the geometrical interpretation of the action of $\mathbf{g}^{*}$ on functions on $G$. We thus have proven the second of the main theorems. We end this section with the following simple observation.

Lemma (4.11). Let $V$ be a representation of $\mathbf{g}^{*} \cong \mathbf{a}_{\mathbf{c}} \oplus \mathbf{n}_{\mathbf{c}}$, then $H_{*}\left(\mathbf{g}^{*}, V\right)$ is the total homology of the following double complex

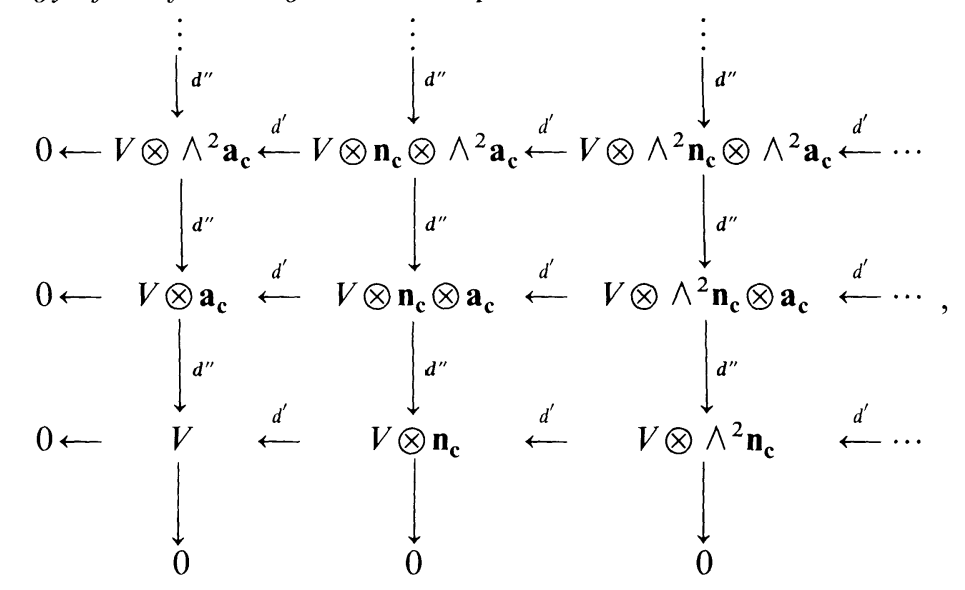

where

$$
d^{\prime}=d_{\left(n_{\mathbf{c}}, V\right)} \otimes 1, \quad d^{\prime \prime}=(-1)^{*} d_{\left(\mathbf{a}_{\mathbf{c}}, V \otimes \wedge^{*} \mathbf{n}_{\mathbf{c}}\right)},
$$

$d_{(\mathbf{g}, V)}$ denotes the standard boundary operator for Lie algebra homology as defined in (4.9), and $\mathbf{a}_{\mathbf{c}}$ acts on $\wedge^{*} \mathbf{n}_{\mathbf{c}}$ by ad.

This is due to the fact that $\mathbf{g}^{*}$ is the semi-direct product of $\mathbf{a}_{\mathbf{c}}$ and $\mathbf{n}_{\mathbf{c}}$. The proof is straightforward. 


\section{The Lie Algebra Homology}

In the previous section we have identified the $E^{2}$ of the spectral sequence leading to the Hochschild homology of quantum groups with the Lie algebra homology of $\mathbf{g}^{*}$ with coefficients in $A_{0}$. In this section we will compute this Lie algebra homology. Here we will have to treat the formal and algebraic functions separately.

5.1. Formal Functions. Recall that we have the identification:

$$
\mathbf{C}^{f}\left(K_{\mathbf{C}}\right) \cong\left\{f \in \mathbf{C}^{f}\left(G_{\mathbf{C}}\right) \mid f\left(g e^{X}\right)=e^{\rho_{\mathbf{a}}(X)} f(g), \forall g \in G_{\mathbf{C}}, X \in \mathbf{g}^{*}\right\}
$$

on which $\mathbf{g}$ and $\mathbf{g}^{*}$ act by infinitesimal left translation $l$. $l$ extends naturally to a left action of $U(\mathbf{g})$ on $\mathbf{C}^{f}\left(K_{\mathbf{C}}\right)$, and the pairing

$$
\langle x, f\rangle=(l(x) f)(e), \quad \forall x \in U(\mathbf{g}), \quad f \in \mathbf{C}^{f}\left(K_{\mathbf{C}}\right)
$$

identifies $\mathbf{C}^{f}\left(K_{\mathbf{C}}\right)$ as the dual of $U(\mathbf{g})$. The induced Hopf algebra structure on $\mathrm{C}^{f}\left(K_{\mathrm{C}}\right)$ is the usual one except the coproduct is $\Delta=\sigma \Delta_{0}$, where $\Delta_{0}$ is the usual coproduct and $\sigma$ is the permutation (had we chosen the infinitesimal right translation in defining the pairing the induced coproduct of $\mathbf{C}^{f}\left(K_{\mathbf{C}}\right)$ would have been the usual one).

The universal enveloping algebra is naturally filtered

$$
\{1\}=U(\mathbf{g})_{0} \subset U(\mathbf{g})_{1} \subset U(\mathbf{g})_{2} \subset \cdots \subset U(\mathbf{g})_{\infty}=U(\mathbf{g}),
$$

where $U(\mathbf{g})_{n}$ consists of those which involve product of no more than $n$ elements of g. This induces a natural filtration on the dual space $\mathbf{C}^{f}\left(K_{\mathbf{C}}\right)$,

$$
\mathbf{C}^{f}\left(K_{\mathbf{C}}\right)=C^{f}\left(K_{\mathbf{C}}\right)_{0} \supset \mathbf{C}^{f}\left(K_{\mathbf{C}}\right)_{1} \supset \mathbf{C}^{f}\left(K_{\mathbf{C}}\right)_{2} \supset \cdots \supset \mathbf{C}^{f}\left(K_{\mathbf{C}}\right)_{\infty}=\phi,
$$

where $\mathbf{C}^{f}\left(K_{\mathbf{C}}\right)_{n}$ vanishes on $U(\mathbf{g})_{n-1}$.

Proposition (5.1.1). $\forall y \in \mathbf{g}^{*}, f \in \mathbf{C}^{f}\left(K_{\mathbf{C}}\right)_{n}$, we have $\sigma(y) f \in \mathbf{C}^{f}\left(K_{\mathbf{C}}\right)_{n}$. Therefore the complex $C \cdot\left(\mathbf{g}^{*}, \mathbf{C}^{f}\left(K_{\mathbf{C}}\right)\right)$ is a filtered complex.

Proof. $\forall x \in U(\mathbf{g})_{n}$, we want to show that $\langle x, \sigma(y) f\rangle=0$. Note that $\sigma=l+\rho_{\mathrm{a}}$ and $l$ actually extends to a left action of $U\left(\mathbf{g}_{\mathbf{c}}\right)$ of $V$. Since $x, y \in U\left(\mathbf{g}_{\mathbf{c}}\right)$ and $(l(y) f)(e)=(-r(y) f)(e)=-\rho_{\mathbf{a}}(y) f(e)$, we have:

$$
\langle x, \sigma(y) f\rangle=\left\langle x,\left(l(y)+\rho_{\mathrm{a}}(y)\right) f\right\rangle=l(x y) f(e)+\rho_{\mathbf{a}}(y) l(x) f(e)=l(x y) f(e),
$$

where the second term is zero by assumption. Note that for formal functions the constant term is unimportant while for algebraic functions that term is vital. Suffice to show $l(x y) f(e)=0$ for $x=x_{1} x_{2} \cdots x_{n}$, with $x_{j} \in \mathbf{g}$. Recall $\left[x_{j}, y\right]=\operatorname{ad}_{y}^{*}\left(x_{j}\right)-\operatorname{ad}_{x_{j}}^{*}(y)$. We use this to move $y \in \mathbf{g}^{*}$ to the left in $l\left(x_{1} x_{2} \cdots x_{n} y\right)$. Whenever $y^{\prime} \in \mathbf{g}^{*}$ appears on the far left we can replace it by a number $\left(-\rho_{\mathbf{a}}\left(y^{\prime}\right)\right)$. It is clear that we eventually have $l(x y) f(e)=l\left(x_{y}\right) f(e)$ and $x_{y} \in U(\mathbf{g})_{n}$. Thus $l(x y) f(e)=0$. Also it is easy to see that the leading term of $x_{y}$ is $\operatorname{ad}_{y}^{*}(x)$.

As a corollary of the above proof we have:

Corollary (5.1.2). For $f \in \mathbf{C}^{f}\left(K_{\mathbf{C}}\right)_{n} / \mathbf{C}^{f}\left(K_{\mathbf{C}}\right)_{n+1}, x \in U(\mathbf{g})_{n}$, we have

$$
\langle x, \sigma(y) f\rangle=\left\langle\operatorname{ad}_{y}^{*}(x), f\right\rangle \text {. }
$$

Note that $\mathbf{C}^{f}\left(K_{\mathbf{C}}\right)_{n} / \mathbf{C}^{f}\left(K_{\mathbf{C}}\right)_{n+1} \cong\left(U(\mathbf{g})_{n} / U(\mathbf{g})\right)^{\prime} \cong\left(S^{n}(\mathbf{g})\right)^{\prime} \cong S^{n}\left(\mathbf{g}^{*}\right)$, where $S^{n}$ 
denotes the $n^{\text {th }}$ symmetric power. Therefore the $E^{1}$ of the spectral sequence of the filtered complex $C .\left(\mathbf{g}^{*}, \mathbf{C}^{f}\left(K_{\mathbf{C}}\right)\right)$ is $H\left(\mathbf{g}^{*}, S^{n}\left(\mathbf{g}^{*}\right)\right)$, where $\mathbf{g}^{*}$ acts on $S^{n}(\mathbf{g})$ by ad.

Proposition (5.1.3).

$$
H_{p}\left(\mathbf{g}^{*}, S^{n}\left(\mathbf{g}^{*}\right)\right) \cong S^{n}\left(\mathbf{a}_{\mathbf{c}}\right) \otimes \wedge^{p} \mathbf{a}_{\mathbf{c}} .
$$

Proof. We compute this Lie algebra homology by the double complex as in Lemma (4.11). Note that $\mathbf{a}_{\mathbf{c}}$ acts on both $\Lambda^{*} \mathbf{n}_{\mathbf{c}}$ and $S^{n}\left(\mathbf{g}^{*}\right)$ semisimply with non-positive weight. Thus if we compute the $\mathbf{a}_{\mathbf{c}}$-homology first we immediately see that the spectral sequence is degenerate at the first term and yields the above result.

Note that we have a natural morphism of the filtered complexes

$$
\phi: C .\left(\mathbf{C}^{f}\left(K_{\mathbf{C}}\right), \mathbf{g}^{*}\right) \rightarrow C .\left(\mathbf{C}^{f}\left(T_{\mathbf{C}}\right), \mathbf{a}_{\mathbf{c}}\right),
$$

which induces a morphism of the associated spectral sequences

$$
\phi_{*}: E^{n}\left(\mathbf{g}^{*}\right) \rightarrow E^{n}\left(\mathbf{a}_{\mathbf{c}}\right) \text {. }
$$

The above proposition simply says that $\phi$ induces an isomorphism at $E^{1}$, so it also induces an isomorphism at $E^{\infty}$. Note that all the filtrations considered in this subsection are complete, thus we can apply the comparison theorem (see, for example, [20] chapter 8) to conclude

Theorem (5.1.4).

$$
H_{n}\left(\mathbf{g}^{*}, \mathbf{C}^{f}\left(K_{\mathbf{C}}\right)\right) \cong \mathbf{C}^{f}\left(T_{\mathbf{C}}\right) \otimes \wedge^{n} \mathbf{a}_{\mathbf{c}} \cong \Omega_{f}^{n}\left(T_{\mathbf{C}}\right) .
$$

Note that we have completeness of filtration in (5.4) because we are dealing with formal functions. The analogous statement is false for algebraic functions. This is the reason for us to treat formal and algebraic functions separately.

5.2. Algebraic Functions. Now we do not have a nice filtration for $\mathbf{C}\left[K_{\mathbf{C}}\right]$ as we have for $\mathbf{C}^{f}\left(K_{\mathbf{C}}\right)$, so we have to look for other ways to compute the Lie algebra homology for algebraic functions. First of all we notice the following

Lemma (5.2.1). $\sigma$ and $\sigma_{0}$ commute with the right translation by the Cartan subalgebra of $\mathbf{g}$.

Proof. Right translation corresponds to the action of left invariant vector field. $\forall H \in \mathbf{h}$, notice that $\left[H^{l}, \omega_{r}\right]=0$ and $\operatorname{ad}_{H}(R)=0$, it is obvious that we have $\left[H^{l}, \sigma\left(J^{a}\right)\right]=0$. This should also be obvious from the geometric interpretation of the actions $\sigma$ and $\sigma_{0}$.

For algebraic functions on $K_{\mathbf{C}}$, the infinitesimal right action of the Cartan subalgebra acts semi-simply, with the eigenvalues ranging over the weight lattice $\Lambda$ of $(\mathbf{g}, \mathbf{h})$. We have the decomposition

$$
\mathbf{C}\left[K_{\mathbf{C}}\right]=\bigoplus_{\lambda \in \Lambda} V_{\lambda},
$$

and because of the previous lemma we have

$$
H_{n}\left(\mathbf{g}^{*}, \mathbf{C}\left[K_{\mathbf{C}}\right]\right)=\bigoplus_{\lambda \in \Lambda} H_{n}\left(\mathbf{g}^{*}, V_{\lambda}\right) .
$$


We can identify $V_{\lambda}$ as

$$
V_{\lambda} \cong\left\{f \in \mathbf{C}^{a}\left(G_{\mathbf{C}}\right) \mid f\left(g e^{X}\right)=e^{-\left(\lambda-\rho_{\mathbf{a}}\right)(X)} f(g), \forall g \in G_{\mathbf{C}}, X \in \mathbf{h}_{\mathbf{c}} \oplus \mathbf{n}_{\mathbf{c}}\right\},
$$

where $\mathbf{h}_{\mathbf{c}}=\mathbf{t}_{\mathbf{c}} \oplus \mathbf{a}_{\mathbf{c}}, \mathbf{t}_{\mathbf{c}}$ is the Cartan subalgebra of $\mathbf{k}_{\mathbf{c}} \cong \mathbf{g}, \lambda$ is a linear functional on $\mathbf{t}_{\mathbf{c}}$ extending trivially to that on $\mathbf{h}_{\mathbf{c}} \oplus \mathbf{n}_{\mathbf{c}}, \rho_{\mathbf{a}}$ is a linear functional on $\mathbf{a}_{\mathbf{c}} \oplus \mathbf{n}_{\mathbf{c}} \cong \mathbf{g}^{*}$ extending to that on $\mathbf{h}_{\mathbf{c}} \oplus \mathbf{n}_{\mathbf{c}} ; \mathbf{g}^{*}$ acts on $V_{\lambda}$ by $\sigma=\sigma_{0}+\rho_{\mathbf{a}}$ and $\sigma_{0}$ has been identified with the infinitesimal left translation.

We notice the close relationship between our $V_{\lambda}$ and induced representation [25] for real Lie group $G$. Let $U_{\lambda}$ be a $\left(T, \mathbf{a}_{\mathbf{c}}\right)$-module such that $T$ acts by $e^{\lambda}$ and $\mathbf{a}_{\mathbf{c}}$ acts trivially. The induced representation for $G$ is defined as

$$
\operatorname{Ind}_{T A N}^{G}\left(U_{\lambda}\right)=\left\{f: G \stackrel{\text { continuous }}{\longrightarrow} \mathbf{C} \mid f\left(g e^{X}\right)=e^{-\left(\lambda-\rho_{\mathbf{a}}\right)(X)} f(g), \forall g \in G, X \in \mathbf{h} \oplus \mathbf{n}\right\},
$$

where $\lambda$ is real linear on $\mathbf{t}$ and complex linear on $\mathbf{t}_{\mathbf{c}}$, extends naturally to $\mathbf{h}_{\mathbf{c}} \oplus \mathbf{n}_{\mathbf{c}} ; \rho_{\mathbf{a}}$ is real linear on a and complex linear on $\mathbf{a}_{\mathbf{c}}$, extends naturally to $\mathbf{h}_{\mathbf{c}} \oplus \mathbf{n}_{\mathbf{c}}$; and $G$ acts by left translation

$$
(g f)(h)=f\left(g^{-1} h\right), \quad \forall g, h \in G .
$$

The Lie algebra of $G$ acts by infinitesimal left translation, which extends naturally to an action of the complexified Lie algebra $\mathbf{g}_{\mathbf{c}}$. Note in (5.12) the minus sign in front of $\rho_{\mathbf{a}}$ is due to the fact that our $\mathbf{n}_{\mathbf{c}}$ corresponds to the negative restricted roots.

Here we use $T A N$ to denote the minimal parabolic subgroup instead of the standard $M A N$ since we want to emphasize the fact that $M:=Z_{K}\left(\mathbf{a}_{\mathbf{c}}\right)=T$ (because $G$ is also complex). Since $G, K$ are real forms of $G_{\mathbf{C}}, K_{\mathbf{C}}$ we have natural homomorphism from $V_{\lambda}$ to $\operatorname{Ind}_{T A N}^{G}\left(U_{\lambda}\right)$. Actually $V_{\lambda}$ is identified with the Harish-Chandra module $[18,25]$ of $\operatorname{Ind}_{T A N}^{G}\left(U_{\lambda}\right)$.

We thus have reduced the computation of the $E^{2}$ of the spectral sequence converging to the Hochschild homology of the quantum algebraic groups to the computation of Lie algebra homology of $\mathbf{a}_{\mathbf{c}} \oplus \mathbf{n}_{\mathbf{c}}$ with coefficient in Harish-Chandra modules $V_{\lambda}$ for $\lambda \in \Lambda$ the weight lattice of $\left(\mathbf{k}_{\mathbf{c}}, \mathbf{t}_{\mathbf{c}}\right)$. We will also think of $\lambda$ as a linear functional on $\mathbf{h}_{\mathbf{c}}=\mathbf{t}_{\mathbf{c}} \oplus \mathbf{a}_{\mathbf{c}}$ with $\left.\lambda\right|_{\mathbf{a}_{\mathbf{c}}}=0$. As for the formal functions we will use the double complex (4.22) to compute the Lie algebra homology, but this time we compute the $\mathbf{n}_{\mathbf{c}}$-homology first. The $\mathbf{n}_{\mathbf{c}}$-homology of Harish-Chandra modules has been extensively studied for its importance in representation theory $[6,18]$. But unfortunately the $\mathbf{n}_{\mathbf{c}}$-homologies of Harish-Chandra modules are not explicitly known in general. What makes life much easier for us is the following. First of all $G$ has a complex structure $G \cong K_{C}$ and $T A N$ is a minimal parabolic subgroup. Secondly we only consider those induced representations $\operatorname{Ind}_{T A N}^{G}\left(U_{\lambda}\right)$ such that $\mathbf{a}_{\mathbf{c}}$ acts on $U_{\lambda}$ trivially. As we will see this simplifies the computation a great deal. Thirdly even in our special cases we do not need a full knowledge of $\mathbf{n}_{\mathbf{c}}$-homology of Harish-Chandra modules. $H_{*}\left(\mathbf{n}_{\mathbf{c}}, V_{\lambda}\right)$ is only the $E^{1}$ term of the spectral sequence associated with the double complex (4.22), its $E_{n}^{2}$ term is $H_{n}\left(\mathbf{a}_{\mathbf{c}}, H \cdot\left(\mathbf{n}_{\mathbf{c}}, V_{\lambda}\right)\right)$, so we may forget about those of $\mathbf{n}_{\mathbf{c}}$-homology on which $\mathbf{a}_{\mathbf{c}}$ acts with non-zero (generalized) eigenvalues.

Let us first recall some facts about Harish-Chandra modules.

Lemma (5.2.2) [5]. Any Harish-Chandra module is finitely generated over $U\left(\mathbf{n}_{\mathbf{c}}\right)$. 
Lemma (5.2.3) [18]. The $\mathbf{n}_{\mathbf{c}}$-homology of any Harish-Chandra module is a Harish-Chandra module for $M A$ (which in our case is $T A$ ).

So $\mathbf{n}_{\mathbf{c}}$-homology of Harish-Chandra module is finite dimensional, and decomposes into Jordan cells under the action of $\mathbf{t}_{\mathbf{c}} \oplus \mathbf{a}_{\mathbf{c}}$. We define the generalized eigenspace with generalized eigenvalue $\mu \in \mathbf{a}_{\mathbf{c}}^{*}$ to be the largest subspace on which $x-\mu(x)$ acts nilpotently for all $x \in \mathbf{a}_{\mathbf{c}}$. Note $T$ acts semisimply on $\mathbf{C}\left[K_{\mathbf{C}}\right]$ and $V_{\lambda}$, thus also acts semisimply on $H_{*}\left(\mathbf{n}_{\mathbf{c}}, V_{\lambda}\right)$. But in general $\mathbf{a}_{\mathbf{c}}$ does not act semisimply on $V_{\lambda}$ so we expect some non-trivial Jordan cells in $H_{*}\left(\mathbf{n}_{\mathbf{c}}, V_{\lambda}\right)$ with respect to the action of $\mathbf{a}_{\mathbf{c}}$.

Definition (5.2.4) [18]. Let $V$ be a Harish-Chandra module for $G$, then $v \in \mathbf{a}_{\mathbf{c}}^{*}$ is a homology exponent of $H_{*}\left(\mathbf{n}_{\mathbf{c}}, V\right)$ if $H_{*}\left(\mathbf{n}_{\mathbf{c}}, V\right)_{v} \neq 0$, where

$$
H_{*}\left(\mathbf{n}_{\mathbf{c}}, V\right)_{v}=\text { generalized }\left(v-\rho_{\mathbf{a}}\right) \text { eigenspace of } H_{*}\left(\mathbf{n}_{\mathbf{c}}, V\right) \text {. }
$$

A leading homology exponent is one that cannot be expressed as the sum of another homology exponents and a non-zero sum of negative restricted roots, i.e., leading homology exponents are the largest ones with respect to the obvious partial order induced by the positive restricted roots.

The shift by $\rho_{\mathbf{a}}$ is to make the notation compatible with Harish-Chandra's labelling of the infinitesimal characters of $Z\left(\mathbf{g}_{\mathbf{c}}\right)$, the minus sign in front of $\rho_{\mathbf{a}}$ is determined by the fact that our $\mathbf{n}_{\mathbf{c}}$ corresponds to the negative restricted roots.

Note that we are only interested in those of $H_{*}\left(\mathbf{n}_{\mathbf{c}}, V\right)$ on which $\mathbf{a}_{\mathbf{c}}$ acts nilpotently via $\sigma=\sigma_{0}+\rho_{\mathbf{a}}$, i.e., $\mathbf{a}_{\mathbf{c}}$ acts by generalized eigenvalue $\left(-\rho_{\mathbf{a}}\right)$ via $\sigma_{0}$. Recall that $\sigma_{0}$ is the natural geometrical action, thus we are only interested in the homology exponent zero.

Lemma (5.2.5) (Schmid's Vanishing Theorem $[18,38])$. Let $v$ be a leading homology exponents, then $H_{n}\left(\mathbf{n}_{\mathbf{c}}, V\right)_{v}=0$, unless $n=0$.

The best we can hope now is that zero is the leading homology exponent. Then only $H_{0}\left(\mathbf{n}_{\mathbf{c}}, V_{\lambda}\right)$ contributes to the $\mathbf{a}_{\mathbf{c}}$-homology, therefore the spectral sequence of the double complex (4.22) is degenerate at $E^{2}$, i.e.,

$$
H_{n}\left(\mathbf{a}_{\mathbf{c}} \oplus \mathbf{n}_{\mathbf{c}}, V_{\lambda}\right)=E_{n}^{\infty} \cong E_{n}^{2}=H_{n}\left(\mathbf{a}_{\mathbf{c}}, H_{0}\left(\mathbf{n}_{\mathbf{c}}, V_{\lambda}\right)\right) \cong \mathbf{C}^{l(\lambda)} \otimes \wedge^{n} \mathbf{a}_{\mathbf{c}}
$$

where $l(\lambda)$ is the number of irreducible Jordan cells in $H_{0}\left(\mathbf{n}_{\mathbf{c}}, V_{\lambda}\right)_{0}$. Actually zero is a leading exponent (thus the unique one) as a consequence of the following lemma, which also gives the dimension of $H_{0}\left(\mathbf{a}_{\mathbf{c}}, V_{\lambda}\right)_{0}$.

Lemma (5.2.6) (Osborne's Conjecture [18]). Let $V$ be a Harish-Chandra module of $G, \Theta_{G}(V)$ the global character, then

$$
\left.\Theta_{G}(V)\right|_{(T A)^{-} \cap G^{\prime}}=\left.\frac{\sum_{p}(-1)^{p} \Theta_{T A}\left(H_{p}\left(\mathbf{n}_{\mathrm{c}}, V\right)\right)}{\sum_{p}(-1)^{p} \Theta_{T A}\left(\wedge^{p} \mathbf{n}_{\mathrm{c}}\right)}\right|_{(T A)^{-} \cap G^{\prime}},
$$

where. $G^{\prime}$ is the set of the regular semisimple elements of $G .(T A)^{-}$is specified as follows: 
$(T A)^{-}=$interior, in $T A$, of the set

$$
\left\{g \in T A \mid \sum_{p}(-1)^{p} \Theta_{T A}\left(\wedge^{p} \mathbf{n}_{\mathbf{c}}\right)(g a) \geqq 0, \forall a \in A^{-}\right\},
$$

where $A^{-}$is the negative Weyl chamber cut out by the positive restricted root system. $A^{-}=\left\{a \in A \mid e^{\alpha}(a)<0, \forall \alpha \in \Phi^{+}\left(\mathbf{g}_{\mathbf{c}}, \mathbf{a}_{\mathbf{c}}\right)\right\}$.

The restriction to $G^{\prime}$ is to avoid certain singularities in the global character, and the restriction to $(T A)^{-}$is to avoid complicated sign conventions. Actually every $T A$-character is completely determined by its restriction to $(T A)^{-} \cap G^{\prime}$. We remind the reader that the above formulation is for our case in which $G$ has a complex structure. In general one should write $M A$ in the place of $T A$.

The global character of induced representations is known. See, for example, $[25,45]$. It is especially simple in our case.

$$
\Theta_{G}\left(V_{\lambda}\right)(t a)=\frac{\sum_{w \in W} e^{w \lambda} \otimes 1}{\sum_{p}(-1)^{p} \Theta_{T A}\left(\wedge^{p} \mathbf{n}_{\mathrm{c}}\right)}(t a),
$$

where $\operatorname{ta} \in(T A)^{-} \cap G^{\prime}, W$ the Weyl group of $\left(\mathbf{k}_{\mathbf{c}}, \mathbf{t}_{\mathbf{c}}\right)$.

One immediately sees that zero is the leading homology exponent. Indeed, according to Schmid's vanishing theorem there is no cancellation in the alternating sum on the right-hand side of (5.16) for the leading homology exponents, so they will show up on the right-hand side. But zero is the only exponent one can count on the left-hand side of (5.16). Another immediate consequence is

$$
\operatorname{dim}\left(H_{0}\left(\mathbf{n}_{\mathbf{c}}, V_{\lambda}\right)_{0}\right)=|W|=\text { the order of the Weyl group. }
$$

Therefore when $\lambda$ is regular, i.e., $w_{1} \lambda=w_{2} \lambda$, if and only if $w_{1}=w_{2}$, there are no non-trivial Jordan cells and we get $l(\lambda)=|W|$. When $\lambda$ is singular there might be non-trivial Jordan cells and we will see there are. The last lemma we are going to quote from representation theory is the Frobenius reciprocity theorem which will help us pin down $H_{0}\left(\mathbf{n}_{\mathbf{c}}, V_{\lambda}\right)_{0}$.

Lemma (5.2.7) (Frobenius Reciprocity Theorem [5]). Let $V$ be a Harish-Chandra module for $G, U_{\sigma, v}$ be a $\left(T, \mathbf{a}_{\mathbf{c}}\right)$-module such that $T$ acts by character $\sigma$ and $\mathbf{a}_{\mathbf{c}}$ acts by $v \in \mathbf{a}_{\mathbf{c}}^{*}$. Then we have

$$
\operatorname{Hom}_{\left(K, \mathbf{g}_{c}\right)}\left(V, \operatorname{Ind}_{T A N}^{G}\left(U_{\sigma, v}\right)\right) \cong \operatorname{Hom}_{\left(T, \mathbf{t}_{\mathrm{c}} \oplus \mathbf{a}_{\mathrm{c}}\right)}\left(H_{0}\left(\mathbf{n}_{\mathrm{c}}, V\right), U_{\sigma, v}\right) \text {. }
$$

The isomorphism in the reciprocity theorem is given as follows. For $\Phi \in \operatorname{Hom}_{\left(K, \mathbf{g}_{\mathrm{c}}\right)}\left(V, \operatorname{Ind}_{T A N}^{G}\left(U_{\sigma, v}\right)\right)$, define

$$
\phi: V \rightarrow U_{\sigma, v}, \quad \phi(v)=\Phi(v)(e), \quad \forall v \in V .
$$

It is easy to see that $\phi \in \operatorname{Hom}_{\left(T, \mathbf{t}_{\mathfrak{c}} \oplus \mathbf{n}_{\mathrm{c}}\right)}\left(H_{0}\left(\mathbf{n}_{\mathfrak{c}}, V\right), U_{\sigma, v}\right)$ and $\Phi$ is uniquely determined by $\phi$.

Note the right-hand side of (5.20) only counts the irreducible Jordan cells of $\mathbf{t}_{\mathbf{c}} \oplus \mathbf{a}_{\mathbf{c}}$, but $\mathbf{t}_{\mathbf{c}}$ acts semisimply so it only counts those of $\mathbf{a}_{\mathbf{c}}$. This is exactly what we are interested in for $\mathbf{a}_{\mathbf{c}}$-homology. Now we need to know the dimension of the space of the intertwining operators between our $V_{\lambda}$ and $\operatorname{Ind}_{T A N}^{G}\left(U_{e^{w \lambda}, 0}\right), w \in W$. It is known that this is one ([25] Theorem (14.13),(14.43)). So the number of the 
irreducible Jordan cells in $H_{0}\left(\mathbf{n}_{\mathbf{c}}, V_{\lambda}\right)_{0}$ is the number of the elements in the orbit generated by $W$ through $\lambda$ in the weight lattice $\Lambda$. This number $l(\lambda)$ is smaller than $|W|$ when $l$ is singular and there are indeed non-trivial Jordan cells. We thus have the following

\section{Proposition (5.2.8).}

$$
H_{n}\left(\mathbf{g}^{*}, V_{\lambda}\right) \cong \mathbf{C}^{l(\lambda)} \otimes \wedge^{n} \mathbf{a}_{\mathbf{c}}
$$

where $l(\lambda)$ is the number of elements on the orbit generated by Weyl group $W(\mathbf{g}, \mathbf{h})$ through $\lambda$ in the weight lattice $\Lambda(\mathbf{g}, \mathbf{h})$.

Remark. When we identify the $\left(E^{1}, d_{1}\right)$ with the Lie algebra homology in Sect. 4, we find that $\mathbf{g}^{*}$ acts on $\mathbf{C}[G]$ by $\sigma\left(J^{a}\right)=Y^{a}-f^{a}$, where $Y^{a}$ is the usual dressing transformation and $f^{a}$ is a multiplication operator. This $f^{a}$ plays an unimportant role in computation of homology for formal functions. Actually one can replace $\sigma$ with $\sigma^{(\gamma)}\left(J^{a}\right)=Y^{a}-\gamma f^{a}, \gamma \in \mathbf{C}$, and get the same Lie algebra homology. But it is absolutely crucial for the algebraic functions. Different values of $\gamma$ would change the homology drastically. Incidentally the computation is the easiest for $\gamma=1$ since $f^{a}$ provides first of all the right modification for the normalization of induced representations, and secondly the right shift to be compatible with Harish-Chandra's labelling of infinitesimal characters. The first is responsible for our representations being induced from $U_{\sigma, v}$ with $v=0$. The second enables us to concentrate on the homology exponent zero.

Let $H=T A$ be the complex Cartan subgroup of $K_{\mathrm{C}}, N_{G}$ be the normalizer of $H$ in $K_{\mathrm{C}} \cong G$. For its close resemblance to the functions on the normalizer, we denote

$$
\tilde{\mathbf{C}}\left[N_{G}\right]=\bigoplus_{\lambda \in \Lambda} \mathbf{C}^{l(\lambda)}, \tilde{\Omega}_{n}\left[N_{G}\right]=\tilde{\mathbf{C}}\left[N_{G}\right] \otimes \wedge^{n} \mathbf{a}_{\mathbf{c}} .
$$

As a corollary of the previous proposition we get the main result of this subsection.

Theorem (5.2.9).

$$
H_{n}\left(\mathbf{g}^{*}, \mathbf{C}\left[K_{\mathbf{C}}\right]\right) \cong \tilde{\mathbf{C}}\left[N_{G}\right] \otimes \wedge^{n} \mathbf{a}_{\mathbf{c}} \cong \tilde{\Omega}_{n}\left[N_{G}\right] .
$$

We thus obtain the Lie algebra homology for the algebraic case by applying a series of results from representation theory. Note the answer is different from that of the formal case. There we get differential forms on the unquantized maximal torus (with formal coefficients of course), here we have those differential forms (with algebraic coefficients) which are closely related to the normalizer of $T_{\mathbf{C}}$ in $K_{\mathbf{C}}$.

The Lie algebra homology is the $E^{2}$ of the spectral sequence of Sect. 4 which converges to the Hochschild homology of quantum groups. The convergence problem will be addressed in the next section.

\section{Hochschild and Cyclic Homology of Quantum Groups}

In this section we show that the spectral sequence leading to the Hochschild homology of quantum groups is degenerate at $E^{2}$. We take this as evidence that the properties of quantum groups are determined by the quasiclassical limit since $E^{2}$ is the homology of the Brylinski complex associated with the Poisson Lie group 
$(G, \pi)$. We also compute the cyclic homology in this section, which is relatively easy after we obtain the Hochschild homology. Note $K_{\mathrm{C}}=G$.

6.1. Quantum Formal Groups. It is well known that in the quantized universal enveloping algebra $U_{h}(\mathrm{~g})$ the Cartan subalgebra is not quantized, i.e., there is a natural Hopf algebra homomorphism

$$
\psi: U\left(\mathbf{h}_{\mathbf{c}}\right) \otimes_{\mathbf{C}} \mathbf{C}((h)) \rightarrow U_{h}(\mathbf{g}) .
$$

This induces a homomorphism of Hopf algebras

$$
\Psi: \mathbf{C}^{f}(G) \rightarrow \mathbf{C}^{f}(H) \otimes_{\mathbf{C}} \mathbf{C}((h)),
$$

and morphism between the complexes (2.16) for their Hochschild homologies, thus also induces morphisms between the associated spectral sequences. For $\mathbf{C}^{f}(H) \otimes_{\mathbf{C}} \mathbf{C}((h))$ the spectral sequence is trivial and we have $E^{1} \cong E^{2} \cong \cdots \cong$ $E^{\infty} \cong \Omega_{f}(H)$, where $\Omega_{f}$ denotes the differential forms with formal coefficients. Proposition (5.1.4) shows the $\Psi$ induces an isomorphism for the second term in the spectral sequence. Note the filtration induced by the Planck constant is complete because we are considering formal deformation (i.e., the value of parameter in quantum group is generic). By comparison theorem we have the following

Theorem (6.1.1). $\Psi$ induces an isomorphism for the Hochschild homology, i.e.,

$$
H H_{n}\left(\mathbf{C}_{h}^{f}(G)\right) \cong \Omega_{f}^{n}(H) \otimes \mathbf{C}((h)),
$$

where $H$ is the Cartan subgroup of $G$.

The Hochschild homology may be considered as the $E^{1}$ term of the spectral sequence associated with the double complex for the cyclic homology. The above theorem shows that $\Psi$ induces an isomorphism for the $E^{1}$ term. Again by comparison theorem we have

Theorem (6.1.2). $\Psi$ induces an isomorphism for the cyclic homology between $\mathbf{C}_{h}^{f}(G)$ and $\mathbf{C}^{f}(H) \otimes \mathbf{C}((h))$, i.e.,

$$
H C_{n}\left(\mathbf{C}_{h}^{f}(G)\right) \cong\left(\Omega_{f}^{n}(H) / d \Omega_{f}^{n-1}(H) \oplus H C_{n}(\mathbf{C})\right) \otimes \mathbf{C}((h)), \quad n=0,1,2, \ldots,
$$

where $H$ is the Cartan subgroup of $G$.

This concludes our computation for the Hochschild and cyclic homology of quantum formal groups and we find that they are the same as those of the ring of the formal functions on the complex Cartan subgroups.

6.2. Quantum Algebraic Groups. This subsection is mainly devoted to the proof ${ }^{2}$ that the spectral sequence leading to the Hochschild homology is also degenerate at $E^{2}$ for the algebraic case.

In the formal case the $E^{2}$ term turns out to be that of the Cartan subgroup. Since the Cartan subgroup is unquantized, the comparison theorem immediately yields the Hochschild and cyclic homology in the formal case. But now the $E^{2}$

\footnotetext{
${ }^{2}$ The proof in our first draft was incomplete, as was pointed to us independently by J. Block and E. Getzler
} 
term is related to the normalizer of the Cartan subgroup. Since there is no unquantized normalizer in quantum algebraic groups, it is not obvious that we have degeneracy at $E^{2}$ as in the formal case. However one does expect the spectral sequence associated with the Planck constant to be degenerate at $E^{2}$. As the philosophy of quantization goes, everything should be determined by its quasiclassical limit, in other words, nothing new happens beyond the first term in the deformation. In terms of spectral sequence the quasiclassical limit is taken care of by $\left(E_{*}^{1}, d_{1}\right)$. If nothing is new beyond that, one expects that the spectral sequence is degenerate at $E^{2}$.

We first reduce the proof of $E_{n}^{2} \cong E_{n}^{\infty}$ to that of $E_{0}^{2} \cong E_{0}^{\infty}$. The following lemma is a special case of a Grothendieck theorem about the superposition of functors.

Lemma (6.2.1). Let $A, B$ be associative algebras, $M^{\prime}$ left $A$-module, $M$ right $A$-module; $\phi: A \rightarrow B$ algebra homomorphism. Then there is a spectral sequence with

$$
\begin{aligned}
E_{i j}^{2} & =\operatorname{Tor}_{i}^{B}\left(\operatorname{Tor}_{j}^{A}(M, B), M^{\prime}\right), \\
E_{i j}^{\infty} & =\operatorname{Tor}_{i+j}^{A}\left(M, M^{\prime}\right),
\end{aligned}
$$

Proof. Consider the following double complex

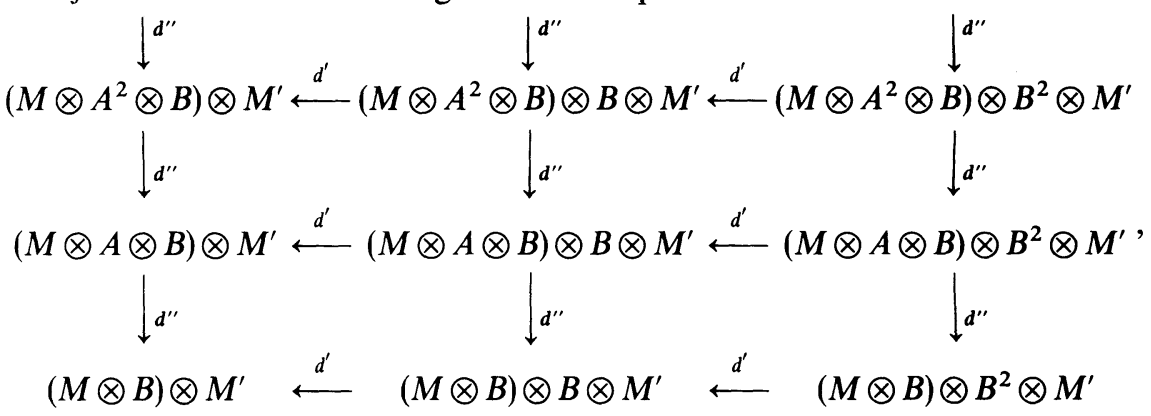

where

$$
d^{\prime}=(-1)^{n} d_{B}\left(M \otimes A^{n} \otimes B, M^{\prime}\right), \quad d^{\prime \prime}=d_{A}(M, B) \otimes 1
$$

and $d_{A}\left(M, M^{\prime}\right)$ denotes the boundary operator of the standard complex for $\operatorname{Tor}_{*}^{A}\left(M, M^{\prime}\right)$. It is easy to check that this really is a double complex. We can compute the associated spectral sequences. If we compute the vertical complex first, we get

$$
E_{i j}^{2}=\operatorname{Tor}_{i}^{B}\left(\operatorname{Tor}_{j}^{A}(M, B), M^{\prime}\right)
$$

Alternatively we can compute the horizontal complex first. Note $M \otimes A^{n} \otimes B$ is a free $B$-module. Therefore

$$
\operatorname{Tor}_{j>0}^{B}\left(M \otimes A^{n} \otimes B, M^{\prime}\right)=0,
$$

and

$$
\operatorname{Tor}_{0}^{B}\left(M \otimes A^{n} \otimes B, M^{\prime}\right)=\left(M \otimes A^{n} \otimes B\right) \otimes{ }_{B} M^{\prime}=M \otimes A^{n} \otimes M^{\prime} .
$$

We see the spectral sequence is degenerate and

$$
E_{i j}^{\infty}=\operatorname{Tor}_{i+j}^{A}\left(M, M^{\prime}\right) \text {. }
$$


From now on, $A=\mathbf{C}_{h}[G], B=\mathbf{C}_{h}[H], \phi: A \rightarrow B$ is induced by the Hopf algebra imbedding $U\left(\mathbf{h}_{\mathbf{c}}\right) \rightarrow U_{h}(\mathbf{g}) . M^{\prime}=\mathbf{C}((h))=k$ as a left $A$-module via the counit $\varepsilon$, $M=\mathbf{C}_{h}[G]$ as a right $A$-module via $\rho$, i.e.,

$$
\rho(a) b=\sum_{j} \tilde{a}_{j}^{s} b a_{j},
$$

where $\Delta(a)=\sum_{j} a_{j} \otimes \tilde{a}_{j}$ and $s$ indicates the antipode. Note as Hopf algebra $\mathbf{C}_{h}[H]=\mathbf{C}[H] \otimes \mathbf{C}((h))$. Recall that $\operatorname{Tor}_{j}^{A}(M, k)$ is the Hochschild homology of the quantum group $\mathbf{C}_{h}[G]$ as proved in Proposition (2.4).

Lemma (6.2.2). Denote the double complex (6.6) by $\mathscr{T} o r_{*}^{B}\left(\mathscr{T} o r_{*}^{A}(M, B), k\right)$ and the standard complex for $\mathrm{Tor}_{*}$ by $\mathscr{T}$ or ${ }_{*}$. There is a quasi-isomorphism of complexes

$$
\mathscr{T}_{*}^{B}\left(\mathscr{T}_{o} r_{*}^{A}(M, B), k\right) \rightarrow \mathscr{T} o r_{*}^{B}\left(M \otimes_{A} B, k\right) \text {. }
$$

Proof. Obviously there is a natural complex map. Note the quasi-classical limit of the double complex (6.6) is exactly the double complex (4.22) that computes the Lie algebra homology $H_{*}\left(\mathbf{g}^{*}, \mathbf{C}[G]\right)$. The lemma simply follows from the fact that the second term of the spectral sequence of the double complex (4.22) $E_{i j}^{2}=H_{i}\left(\mathbf{a}_{\mathbf{c}}, H_{j}\left(\mathbf{n}_{\mathbf{c}}, \mathbf{C}[G]\right)\right)$ is non-vanishing only when $j=0$.

Proposition (6.2.3). The spectral sequence leading to the Hochschild homology of $\mathrm{C}_{h}[G]$ is degenerate at $E^{2}$, i.e.,

$$
H H_{j}\left(\mathbf{C}_{h}[G]\right) \cong H_{j}\left(\mathbf{g}^{*}, \mathbf{C}[G]\right) \otimes \mathbf{C}((h)),
$$

if and only if

$$
H H_{0}\left(\mathbf{C}_{h}[G]\right) \cong H_{0}\left(\mathbf{g}^{*}, \mathbf{C}[G]\right) \otimes \mathbf{C}((h)) .
$$

Proof. By the previous lemma we have two complexes quasi-isomorphic For each complex we can consider the spectral sequence induced by the Planck constant. Obviously their $E^{2}$ terms are isomorphic. So one is degenerate at $E^{2}$ if and only if the other one is. For the complex $\mathscr{T} \operatorname{or}_{*}^{B}\left(M \otimes_{A} B, k\right)$, it is easy to see that the spectral sequence is degenerate at $E^{2}$ if and only if

Thus the lemma.

$$
H_{0}\left(\mathbf{g}^{*}, \mathbf{C}[G]\right) \cong M \otimes_{A} k .
$$

The main result of this subsection is the following Theorem (6.2.4).

$$
H_{0}\left(\mathbf{g}^{*}, \mathbf{C}[G]\right) \otimes_{\mathbf{C}} k \cong M \otimes_{A} k,
$$

where $M=A=\mathbf{C}_{h}[G]$, and $A$ acts on $M$ by $\rho$.

The proof will be divided into a series of lemmas. Proposition (6.2.3) and Theorem (6.2.4) together assert that the spectral sequence induced by the Planck constant for the Hochschild homology is indeed degenerate at $E^{2}$.

In computing the Lie algebra homology $H_{n}\left(\mathbf{g}^{*}, \mathbf{C}[G]\right)$ we use the fact that the $\mathbf{g}^{*}$ action on $\mathbf{C}[G]$ commutes with the infinitesimal right translation by the Cartan subalgebra $\mathbf{h}_{\mathbf{c}}$. We decomposes $\mathbf{C}[G]$ according to this right translation

$$
\mathrm{C}[G]=\bigoplus_{\lambda \in \Lambda} V_{\lambda},
$$


where $\Lambda$ is the weight lattice, and we get

$$
H_{n}\left(\mathbf{g}^{*}, \mathbf{C}[G]\right)=\bigoplus_{\lambda \in \Lambda} H_{n}\left(\mathbf{g}^{*}, V_{\lambda}\right) .
$$

We observe that this decomposition also holds at the quantum level. Recall the Cartan subalgebra is not quantized. We define an action of $U\left(\mathbf{h}_{\mathbf{c}}\right)$ on $M$ in the following way:

$$
r(x)(f)=\sum_{j} f_{j}\left\langle\tilde{f}_{j}, x\right\rangle, \quad \forall f \in M, \quad x \in U\left(\mathbf{h}_{\mathbf{c}}\right),
$$

where $\Delta(f)=\sum_{j} f_{j} \otimes \tilde{f}_{j}$, and $\langle$,$\rangle is the natural paring between \mathbf{C}[G]$ and $U_{h}(\mathbf{g})$. Apparently the classical limit of this action is the infinitesimal right translation. Since $\mathbf{C}_{h}[G]$ is the deformation of $\mathbf{C}[G]$, we have

$$
M=\bigoplus_{\lambda \in \Lambda} M_{\lambda}
$$

The following Lemma asserts that $M_{\lambda}$ is also a right $A$-module via $\rho$.

Lemma (6.2.5). The actions of $U\left(\mathbf{h}_{\mathbf{c}}\right)$ and $\mathbf{C}_{h}[G]$ on $M$ commute.

Proof. Consider arbitrary $H \in \mathbf{h}_{\mathbf{c}}, a \in \mathbf{C}_{h}[G], f \in M$. It follows from the definition that

$$
r(H)\left(f_{1} f_{2}\right)=r(H)\left(f_{1}\right) f_{2}+f_{1} r(H)\left(f_{2}\right) \text {. }
$$

Therefore

$$
r(H) \rho(a)(f)-\rho(a) r(H)(f)=\sum_{j}\left[r(H)\left(\tilde{a}_{j}^{s}\right) f a_{j}+\tilde{a}_{j}^{s} f r(H)\left(a_{j}\right)\right]
$$

where $\Delta(a)=\sum_{j} a_{j} \otimes \tilde{a}_{j}$. Denote

$$
(\Delta \otimes 1) \Delta(a)=(1 \otimes \Delta) \Delta(a)=\sum_{k} b_{k} \otimes c_{k} \otimes d_{k} .
$$

Note for $\mathbf{C}_{h}[G]$ we have $\Delta(s(a))=(s \otimes s) P_{12} \Delta(a)$, where $P_{12}$ is the permutation operator. It then follows that

$$
[r(H), \rho(a)](f)=\sum_{k}\left\langle H, c_{k}^{s}+c_{k}\right\rangle d_{k}^{s} f b_{k}=0 .
$$

As a consequence of the lemma, (6.7) is equivalent to

$$
H_{0}\left(\mathbf{g}^{*}, V_{\lambda}\right) \otimes_{\mathrm{C}} k \cong M_{\lambda} \otimes_{A} k .
$$

Recall that $\left(E_{0}^{2}\right)_{\lambda}=H_{0}\left(\mathbf{g}^{*}, V_{\lambda}\right)$ has dimension $\left|\mathcal{O}_{\lambda}\right|$. We denote the orbit in $\Lambda$ through $\lambda$ generated by the Weyl group $W$ by $\mathcal{O}_{\lambda}$ and the number of distinct weights on the orbit by $\left|\mathcal{O}_{\lambda}\right|$. This provides an upper bound for the dimension of $\left(E_{0}^{\infty}\right)_{\lambda}=M_{\lambda} \otimes_{A} k$. Since $M \otimes_{A} k \simeq A /[A, A]$, we can get a lower bound on that dimension by constructing traces over $A$. The main theorem is proved if we can show that the lower bound is also $\left|\mathcal{O}_{\lambda}\right|$. The following several lemmas are about constructing and counting the traces over $A$.

Lemma (6.2.6). Let $a, b, c, d$ be the generators of $\mathrm{C}_{h}\left[S L_{2}\right]$ as in Example (3.14). There are two families of traces for $\mathbf{C}_{h}\left[S L_{2}\right]$, denoted by $\tau_{n}^{e}, \tau_{n}^{s}$, where $n$ is an integer 
and $n \neq 0$ for $\tau_{n}^{s}$. The following are the only non-zero values of the traces:

(1) $n>0$

$$
\tau_{n}^{e}\left(a^{n+k} d^{k}\right)=1, \quad \tau_{n}^{s}\left(b^{k} c^{n+k}\right)=\frac{(-q)^{k+n}}{1-q^{2 k+n}}, \quad k \geqq 0
$$

(2) $n<0$

$$
\tau_{n}^{e}\left(a^{k} d^{k-n}\right)=1, \quad \tau_{n}^{s}\left(b^{k-n} c^{k}\right)=\frac{(-q)^{k}}{1-q^{2 k-n}}, \quad k \geqq 0
$$

(3) $n=0$

$$
\tau_{0}^{e}\left(a^{k} d^{k}\right)=1, \quad k \geqq 0
$$

where $q=e^{h}$.

We use the elements of the Weyl group $W=\{e, s\}$ to label the families of traces. The naturalness of this notation will become clear later. The proof of this lemma is straightforward and is given in Appendix B. Note the lemma proves the main theorem for $\mathbf{C}_{h}\left[S L_{2}\right]$.

In general, the family of traces associated with the identity of the Weyl group is simply constructed as follows. Recall that the Cartan subalgebra is not quantized. The natural imbedding

$$
U\left(\mathbf{h}_{\mathbf{c}}\right) \otimes_{\mathbf{C}} \mathbf{C}[[h]] \subset U_{h}(\mathbf{g})
$$

induces the Hopf algebra homomorphism

Note

$$
\pi: \mathbf{C}_{h}[G] \rightarrow \mathbf{C}_{h}[H]=\mathbf{C}[H] \otimes_{\mathbf{C}} \mathbf{C}((h)) .
$$

$$
\mathbf{C}[H]=\bigoplus_{\lambda \in \Lambda} \mathbf{C}[H]_{\lambda}
$$

and $\mathbf{C}[H]_{\lambda}$ is one-dimensional. Choose a basis $\left\{f_{\lambda}\right\}$ for $\mathbf{C}[H]$ such that $f_{\lambda} \in \mathbf{C}[H]_{\lambda}$. Let $\left\{f_{\lambda}^{*}\right\}$ be the dual basis. Define

$$
\tau_{\lambda}^{e}=f_{\lambda}^{*} \cdot \pi: \mathbf{C}_{h}[G] \rightarrow k .
$$

This is the family of traces associated with the identity of the Weyl group. We see that the unquantized Cartan subgroup contributes to $E_{0}^{\infty}$.

In quantizing the universal enveloping algebra $[11,21]$, one chooses a Cartan subalgebra and a set of simple roots $\alpha_{1}, \alpha_{2}, \ldots, \alpha_{r}$. For every simple root $\alpha_{j}$, there is a Hopf algebra imbedding

$$
U_{h}\left(s l_{2}\right) \subset U_{h}(\mathbf{g})
$$

which induces a Hopf algebra homomorphism

$$
\pi_{j}: \mathbf{C}_{h}[G] \rightarrow \mathbf{C}_{h}\left[S L_{2}\right] .
$$

Consider an arbitrary element $w$ of the Weyl group. Let $w=s_{i_{1}} s_{i_{2}} \cdots s_{i_{1}}$ be a minimal representation for $w$. Here $l=l(w)$ is the length of $w . s_{j}$ is the simple reflection associated with the simple root $\alpha_{j}$. Define, for arbitrary $\lambda \in \Lambda, n_{1} n_{2} \cdots n_{l} \neq 0$, a linear functional

$$
\tau_{\lambda}^{w}\left(n_{1}, n_{2}, \ldots, n_{l}\right): \mathbf{C}_{h}[G] \rightarrow k
$$


by

$$
\tau_{\lambda}^{w}\left(n_{1}, n_{2}, \ldots, n_{l}\right)=\left(\tau_{n_{1}}^{s} \cdot \pi_{i_{1}} \otimes \cdots \otimes \tau_{n_{l}}^{s} \cdot \pi_{i_{l}} \otimes \tau_{\lambda}^{e}\right) \Delta^{l},
$$

where $\Delta^{l}=(\Delta \otimes 1 \cdots \otimes 1) \cdots(\Delta \otimes 1) \Delta: \mathbf{C}_{h}[G] \rightarrow \mathbf{C}_{h}[G]^{\otimes(l+1)}$.

Lemma (6.2.7). $\tau_{\lambda}^{w}\left(n_{1}, n_{2}, \ldots, n_{l}\right)$ is a trace. It vanishes identically unless

$$
\left.\begin{array}{rl}
n_{l} & =\lambda\left(H_{i_{l}}\right), \\
n_{l-1} & =\lambda\left(s_{i_{l}}\left(H_{i_{l-1}}\right)\right), \\
& \cdots \\
n_{1} & =\lambda\left(s_{i_{l}} s_{i_{l-1}} \cdots s_{i_{2}}\left(H_{i_{1}}\right)\right) .
\end{array}\right\}
$$

When $n_{1}, n_{2}, \ldots, n_{l}$ are thus fixed, $\tau_{\lambda}^{w}=\tau_{\lambda}^{w}\left(n_{1}, n_{2}, \ldots, n_{l}\right)$ takes non-zero values only on $M_{\lambda}$. Different elements of Weyl group give linearly independent traces.

Proof. Since $\Delta, \pi_{j}$ are algebra homomorphisms and $\tau_{\lambda}^{e}, \tau_{n}^{s}$ are traces, (6.16) definitely defines a trace for $\mathbf{C}_{h}[G]$. Note

$$
\tau_{\lambda}^{e}(r(H)(f))=\lambda(H) \tau_{\lambda}^{e}(f), \quad \forall H \in \mathbf{h}_{\mathbf{c}}, \quad f \in M=\mathbf{C}_{h}[G],
$$

we have

$$
\tau_{\lambda}^{w}\left(n_{1}, n_{2}, \ldots, n_{l}\right)(r(H)(f))=\lambda(H) \tau_{\lambda}^{w}\left(n_{1}, n_{2}, \ldots, n_{l}\right)(f) .
$$

Thus $\tau_{\lambda}^{w}$ vanishes on $M_{\lambda^{\prime} \neq \lambda^{\prime}}$.

To get the constraints (6.17), we notice that $U\left(\mathbf{h}_{\mathbf{c}}\right)$ also acts from the left on $\mathbf{C}_{h}[G]$. Explicitly, for any $H \in U\left(\mathbf{h}_{\mathbf{c}}\right), f \in \mathbf{C}_{h}[G]$,

$$
l(H)(f)=\sum_{j}\left\langle f_{j}, H\right\rangle \tilde{f}_{j},
$$

where $\Delta(f)=\sum_{j} f_{j} \otimes \tilde{f}_{j}$. It follows from the definition that

$$
(r(H) \otimes 1) \Delta(f)=(1 \otimes l(H)) \Delta(f)
$$

The constraints on $n_{i_{1}}, \ldots, n_{i_{l}}$ follow from the observation

$$
\tau_{\lambda}^{e}(l(H)(f))=\tau_{\lambda}^{e}(r(H)(f)),
$$

and

$$
\tau_{n}^{s} \cdot \pi_{j}(l(H)(f))=\tau_{n}^{s} \cdot \pi_{j}\left(r\left(s_{j}(H)\right)(f)\right) .
$$

Recall that $\mathbf{C}_{h}[G]$ is spanned by the matrix elements of the finite dimensional representations of $U_{h}(\mathbf{g})$. We always assume that $U\left(\mathbf{h}_{\mathbf{c}}\right)$ is diagonalized in these representations. Since $\tau_{\lambda}^{e}$ is non-zero only on the diagonal matrix elements for which the left and right actions of $U\left(\mathbf{h}_{\mathbf{c}}\right)$ are the same, we get (6.20). To get (6.21), we observe that there is the $q$-analogue of the Weyl group as defined in [24]. Denote the $q$-analogue of $s_{j}$ by $w_{j}$, which generates the $q$-Weyl group. These $w_{j}$ 's act on the representations of $U_{h}(\mathbf{g})$. One can easily check that for $\mathbf{C}_{h}\left[S L_{2}\right]$ the matrix elements with non-zero trace $\tau_{n}^{s}$, i.e., $b^{m} c^{n}$, are on the diagonal when multiplied by the $w$. This gives us

$$
\tau_{n}^{s} \cdot \pi_{j}(l(H)(f))=\tau_{n}^{s} \cdot \pi_{j}\left(r\left(w_{j} H w_{j}^{-1}\right)(f)\right)
$$


But the $q$-Weyl group action on $\mathbf{h}_{\mathbf{c}}$ coincides with that of the ordinary Weyl group, i.e.

$$
w_{j} H w_{j}^{-1}=s_{j}(H) \text {. }
$$

Thus follows (6.21).

It is obvious that $\tau_{\lambda}^{w}$ is not identically zero when $\lambda$ satisfies the constraint (6.17) with $n_{1} \cdots n_{l} \neq 0$. From the above argument, it is also clear that $\tau_{\lambda}^{w}$ is non-zero only on the subspace of $M_{\lambda}$ corresponding to the weight $w(\lambda)$ under the left action of $\mathbf{h}_{\mathbf{c}}$. The linear independence of the traces thus follows immediately.

We have constructed families of traces labeled by the elements of the Weyl group. We remark that they are intrinsically related to the Levendorski-SoibelmanVaksman modules $[41,42,26]$ labeled by the Weyl group and Cartan subgroup. Informally, the relation is as follows. One can take the formal traces from these modules when $|q|<1$. Unfortunately they do not all vanish on the commutant $[A, A]$. The reason is that certain elements of $A=\mathbf{C}_{h}[G]$ are represented by operators which are not of the trace class. What saves the day is that we can use weight space decomposition to pin down the bad components of the formal traces. The good ones are exactly $\tau_{\lambda}^{w}$ with constraints on $\lambda$ as stated in the previous lemma. More on this in Appendix B.

Our next job is to count how many $w \in W$ provide traces to an arbitrary fixed $\lambda \in \Lambda$. The previous lemma tells us what $M_{\lambda}$ will be provided a trace by a particular $w \in W$. Let us first reformulate this in a more geometrical way.

Fix a $\lambda \in \Lambda$, and a minimal representation $w=s_{i_{1}} s_{i_{2}} \cdots s_{i_{l}}$. Let $P_{w}(\lambda)$ denote the ordered set of weights $\left\{\lambda_{l}, \lambda_{l-1}, \ldots, \lambda_{1}, \lambda_{0}\right\}$, where

$$
\lambda_{l}=\lambda, \quad \lambda_{k-1}=\mathrm{s}_{i_{k}}\left(\lambda_{k}\right), \quad k=l, \ldots, 2,1 .
$$

We call this the path associated with the pair $(\lambda, w)$ (with a chosen minimal representation for $w$ ). We say the path has stationary points if $\lambda_{k}=\lambda_{k-1}$ for some $k$.

Lemma (6.2.8). Let $P_{w}(\lambda)$ and $P_{w}^{\prime}(\lambda)$ be two paths corresponding to two choices of minimal representations for $w$. Then $P_{w}(\lambda)$ has stationary points if and only if $P_{w}^{\prime}(\lambda)$ has.

Proof. Let us look at the different parts of the two paths. They come from the non-trivial Coxeter relations

$$
\left(s_{i} s_{j}\right)^{m_{i j}}=e, \quad i \neq j
$$

where

$$
m_{i j}=\frac{\pi}{\arccos \left(\sqrt{a_{i j} a_{j i} / 4}\right)},
$$

and $\left(a_{i j}\right)$ is the Cartan matrix. For any $\lambda$, the sequence $s_{i}(\lambda), s_{j} s_{i}(\lambda), \ldots,\left(s_{i} s_{j}\right)^{m_{i j}}(\lambda)$ form a closed path. Assume $\lambda$ is fixed by $s_{i}$, then it is easy to see that its opposite point $\tilde{\lambda}$ the closed path is fixed by $s_{j}$. If $\tilde{\lambda}$ is a stationary point on $P_{w}(\lambda)$ then either $\tilde{\lambda}$ or the opposite point of $\tilde{\lambda}$ is on $P_{w}^{\prime}(\lambda)$. Thus $P_{w}^{\prime}(\lambda)$ also has stationary points.

We say $w$ is maximal relative to $\lambda$ if $P_{w}(\lambda)$ does not have stationary points. Of course this property is independent of the choice of minimal representation for $w$. 
Corollary (6.2.9). $w \in W$ provides a trace $\tau_{\lambda}^{w}$ for $M_{\lambda}$ if and only if $w$ is maximal relative to $\lambda$.

Proof. From the Lemma (6.2.7), $w$ provides traces to all integral weights except those on the union of the following set of hyperplanes:

$$
\left.\begin{array}{l}
0=\lambda\left(H_{i_{l}}\right), \\
0=\lambda\left(s_{i_{l}}\left(H_{i_{l-1}}\right)\right), \\
\quad \ldots . \\
0=\lambda\left(s_{i_{l}} s_{i_{l-1}} \cdots s_{i_{2}}\left(H_{i_{1}}\right)\right) .
\end{array}\right\}
$$

This is equivalent to

$$
\left\{\begin{array}{l}
0=\left\langle\lambda, \alpha_{i_{l}}\right\rangle, \\
0=\left\langle s_{i_{l}}(\lambda), \alpha_{i_{1-1}}\right\rangle, \\
\cdots \cdots \\
0=\left\langle s_{i_{2}} s_{i_{3}} \cdots s_{i_{l}}(\lambda), \alpha_{i_{1}}\right\rangle,
\end{array}\right.
$$

where $\langle$,$\rangle denotes the inner product in the weight space. The above equations$ mean $\lambda_{k}$ is fixed by $s_{i_{k}}$. The claim is proved.

Lemma (6.2.10). For any $\lambda \in \Lambda$, the number of Weyl group elements that are maximal relative to $\lambda$ equals to $\left|\mathcal{O}_{\lambda}\right|$, the number of points on the orbit $\mathcal{O}_{\lambda}$ generated by the Weyl group $W$ through $\lambda$.

Proof. We first construct a cell complex $\hat{\mathcal{O}}_{\lambda}$ whose vertices are the points on $\mathcal{O}_{\lambda}$. Two distinct vertices are connected by an edge if they are transformed into each other by a simple reflection $s_{j}$. We attach a face to every closed path corresponding to a non-trivial Coxeter relation (6.22) such that the boundary of the face is the closed path. We also choose a base point $\lambda_{0}$ which is either in the fundamental domain (when $\lambda$ is regular) or on the boundary of the fundamental domain (when $\lambda$ is singular). The base point is of course unique for every orbit. We claim that $\hat{\mathcal{O}}_{\lambda}$ is connected and simply connected.

Let us construct another cell complex $\hat{W}$. The vertices are the elements of the Weyl group. There is an edge between $w_{1}$ and $w_{2}$ if $w_{1}=s_{j} w_{2}$ for some $j$. For every closed path corresponding to a non-trivial Coxeter relation, a face is assigned whose boundary is the closed path. We choose the identity $e$ to be the base point. By definition $\hat{W}$ is connected and simply connected. Note the stabilizer subgroup $W_{\lambda_{0}}$ of $\lambda_{0}$ is of the Weyl group type and the associated cell complex $\hat{W}_{\lambda_{0}}$ is connected and simply connected. The homotopy fibration

$$
\hat{W}_{\lambda_{0}} \rightarrow \hat{W} \rightarrow \hat{\mathcal{O}}_{\lambda_{0}}
$$

induces the homotopy exact sequence

$$
\cdots \rightarrow \pi_{1}(\hat{W}) \rightarrow \pi_{1}\left(\hat{\mathcal{O}}_{\lambda_{0}}\right) \rightarrow \pi_{0}\left(\hat{W}_{\lambda_{0}}\right) \rightarrow \pi_{0}(\hat{W}) \rightarrow \pi_{0}\left(\hat{\mathcal{O}}_{\lambda_{0}}\right) \rightarrow 0 .
$$

From this we immediately get

$$
\pi_{1}\left(\hat{\mathcal{O}}_{\lambda_{0}}\right)=0=\pi_{0}\left(\hat{\mathcal{O}}_{\lambda_{0}}\right) .
$$

Thus $\hat{\mathcal{O}}_{\lambda}$ is connected and simply connected.

For any $\lambda \in \Lambda$, the Weyl group can be viewed as a union of cosets $W_{\lambda \lambda^{\prime}}$ labeled 
by the points $\lambda^{\prime} \in \mathcal{O}_{\lambda}$. The elements in $W_{\lambda \lambda^{\prime}}$ transform $\lambda$ to $\lambda^{\prime}$. Since $\mathcal{O}_{\lambda}$ is simply connected, there is only one element in each coset that is maximal relative to $\lambda$. Thus the total number of maximal elements relative to $\lambda$ is $\left|\mathcal{O}_{\lambda}\right|$. The lemma is proved.

Proof of Theorem (6.2.4). We have reduced the proof to that of

$$
H_{0}\left(\mathbf{g}^{*}, V_{\lambda}\right) \otimes_{\mathbf{C}} k \cong M_{\lambda} \otimes_{A} k .
$$

Since $H_{0}\left(\mathrm{~g}^{*}, V_{\lambda}\right)$ has dimension $\left|\mathcal{O}_{\lambda}\right|$, this provides an upper bound for the dimension of $M_{\lambda} \otimes_{A} k$. The above lemma provides a lower bound which is also $\left|\mathcal{O}_{\lambda}\right|$. This means elements of $E_{0}^{2}$ all survive to $E_{0}^{\infty}$. The theorem is proved.

In summarizing, we have

Theorem (6.2.11). The Hochschild homology of the quantum algebraic group is

$$
H H_{n}\left(\mathbf{C}_{h}[G]\right) \cong \tilde{\Omega}_{n}\left[N_{G}\right] \otimes \mathbf{C}((h)),
$$

where $\widetilde{\Omega}_{n}\left[N_{G}\right]$ is defined in (5.22).

The last thing we are going to compute in this paper is the cyclic homology of the quantum algebraic groups. This is now fairly easy when we know the Hochschild homology. Recall $H C_{*}(A)$ is computed by the double complex

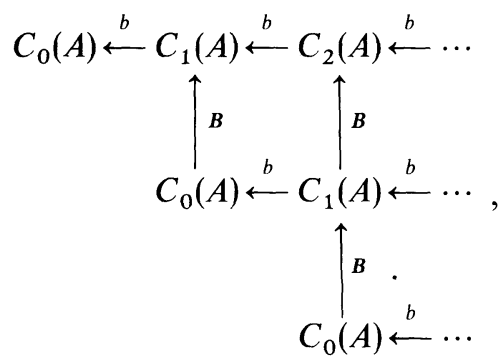

where $b$ and $B$ are defined at the beginning of Sect. 2 .

The Hochschild homology is only the $E^{1}$ term of the spectral sequence associated with the double complex. The complex for $E^{2}$ is

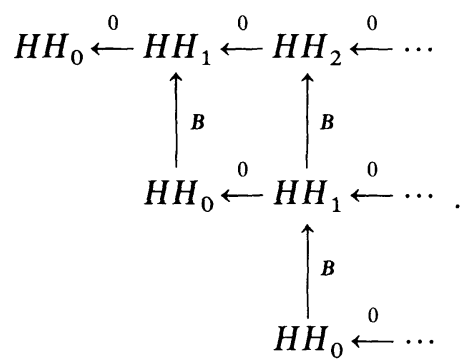

As is well-known from $[10,17]$, the derivation of $r(H), H \in \mathbf{h}_{\mathbf{c}}$ on $A$ induces the operation of Lie derivative

$$
\mathscr{L}_{H}: H H_{n}(A) \rightarrow H H_{n}(A)
$$

and

$$
i_{H}: H H_{n} \rightarrow H H_{n-1}(A)
$$


such that

$$
B \cdot i_{H}+i_{H} \cdot B=\mathscr{L}_{H} .
$$

From this one sees immediately that $B$ is exact on all weight components of $\mathscr{L}_{H}$ except zero. Hence the only contributions to $E^{2}$ (except for the top row in (6.26)) is from $\lambda=0$. Recall

$$
H H_{n}(A) \cong H_{n}\left(\mathbf{g}^{*}, \mathbf{C}[G]\right)=\bigoplus_{\lambda} H_{n}\left(\mathbf{g}^{*}, V_{\lambda}\right) .
$$

Note that $H_{n}\left(\mathbf{g}^{*}, V_{0}\right)$ is one dimensional and is represented by $f=$ constant. So except for the contributions from the top row in (6.26) we can identify the total homology of the double complex (6.26) with the invariant differential forms on the Cartan subgroup $H$. The contribution from the top row is simply $\widetilde{\Omega}_{n}\left[N_{G}\right] / d \widetilde{\Omega}_{n-1}\left[N_{G}\right]$. It is also easy to see the spectral sequence is degenerate at $E^{2}$. The following theorem is now obvious.

Theorem (6.2.12). The cyclic homology for the quantum algebraic group is

$$
H C_{n}\left(\mathbf{C}_{h}[G]\right) \cong\left(\tilde{\Omega}_{n}\left[N_{G}\right] / d \tilde{\Omega}_{n-1}\left[N_{G}\right] \oplus H_{\mathrm{DR}}^{n-2}\left(T_{\mathbf{C}}\right) \oplus H_{\mathrm{DR}}^{n-4}\left(T_{\mathbf{C}}\right) \oplus \cdots\right) \otimes \mathbf{C}((h)),
$$

where $\tilde{\Omega}_{n}\left[N_{G}\right]$ is defined in (5.22).

This concludes our computation for the Hochschild and cyclic homology of quantum algebraic groups. We found that they are determined by the quasiclassical limit.

\section{Appendix A.}

We explain some of our conventions for the calculus on Lie groups.

Let $I_{a}, a=1,2, \ldots, \operatorname{dim} \mathbf{g}$ be the basis for the Lie algebra $\mathbf{g}$, which is identified with the tangent space at the identity $e ; f_{a b}^{c}$ the structure constant, $J^{a}$ the dual basis, $X_{a}^{l}, X_{a}^{r}$ the left, right invariant vector field on $G$ such that $X_{a}^{l}(e)=X_{a}^{r}(e)=I_{a}$; $\omega_{l}^{a}, \omega_{\mathrm{r}}^{a}$ the left, right invariant 1 -form with $\omega_{l}^{a}(e)=\omega_{r}^{a}(e)=J_{a}$. Repeated index always means summation.

First of all,

$$
\begin{aligned}
& {\left[X_{a}^{l}, X_{b}^{l}\right]=f_{a b}^{c} X_{c}^{l},} \\
& {\left[X_{a}^{r}, X_{b}^{r}\right]=-f_{a b}^{c} X_{c}^{r} .}
\end{aligned}
$$

Both $X_{a}^{l}(g)$ and $X_{a}^{r}(g)$ span the tangent space at $g$, they can be transformed into each other as

$$
X_{a}^{l}(g)=\left(\operatorname{Ad}_{g}\right)_{a}^{b} X_{b}^{r}(g), \quad X_{a}^{r}(g)=\left(\operatorname{Ad}_{g^{-1}}\right)_{a}^{b} X_{b}^{l}(g)
$$

and similarly,

$$
\omega_{l}^{a}=\left(\operatorname{Ad}_{g^{-1}}^{*}\right)_{b}^{a} \omega_{r}^{b}, \quad \omega_{r}^{a}=\left(\operatorname{Ad}_{g}^{*}\right)_{b}^{a} \omega_{l}^{b} .
$$

Let $\mathscr{L}_{X}$ denote the Lie derivative corresponding to the vector field $X$, then

$$
\begin{array}{ll}
\mathscr{L}_{X_{a}^{l}} X_{b}^{l}=f_{a b}^{c} X_{c}^{l}, & \mathscr{L}_{X_{a}^{l}} X_{b}^{r}=0, \\
\mathscr{L}_{X_{a}^{r}} X_{b}^{r}=-f_{a b}^{c} X_{c}^{r}, & \mathscr{L}_{X_{a}^{r}}^{X_{b}} X_{b}^{l}=0 ;
\end{array}
$$


and from $\left\langle X_{a}^{l}, \omega_{l}^{b}\right\rangle=\left\langle X_{a}^{r}, \omega_{r}^{b}\right\rangle=\delta_{a}^{b}$, we have

$$
\begin{array}{ll}
\mathscr{L}_{X_{a}^{l}} \omega_{l}^{b}=-f_{a b}^{c} \omega_{l}^{c}, & \mathscr{L}_{X_{a}^{l}} \omega_{r}^{b}=0, \\
\mathscr{L}_{X_{a}^{r}} \omega_{r}^{b}=f_{a b}^{c} \omega_{c}^{r}, & \mathscr{L}_{X_{a}^{r}} \omega_{l}^{b}=0 .
\end{array}
$$

The adjoint action is defined to be

$$
\begin{aligned}
\left\langle I_{b}, \operatorname{ad}_{I_{a}}^{*}\left(J^{c}\right)\right\rangle & =\left\langle\operatorname{ad}_{I_{a}} I_{b}, J^{c}\right\rangle, \text { i.e. } \\
\operatorname{ad}_{I_{a}}^{*}\left(J^{c}\right) & =f_{a b}^{c} J^{b} .
\end{aligned}
$$

The exterior derivative $d$ acts as

$$
\begin{aligned}
d f & =\omega_{l}^{a}\left(X_{a}^{l} f\right)=\omega_{r}^{a}\left(X_{a}^{r} f\right), \\
d \omega_{l}^{a} & =-f_{b c}^{a} \omega_{l}^{b} \wedge \omega_{l}^{c}, \\
d \omega_{r}^{a} & =f_{b c}^{a} \omega_{r}^{b} \wedge \omega_{r}^{c} .
\end{aligned}
$$

The (infinitesimal) left translation of $\mathbf{g}$ on $C^{\infty}(G)$ is

$$
l(I) f(g)=-\mathscr{L}_{X^{r}} f(g)=\lim _{t \rightarrow 0} \frac{1}{t}\left(f\left(e^{-t I} g\right)-f(g)\right) .
$$

\section{Appendix B. Example $G=S L_{2}$}

The quantum algebraic group structure is as discussed in Sect. 3, specifically in Example (3.14). So we will not repeat those definitions.

B.1. The Quasiclassical Limit. We start with the computation of $H_{n}\left(\mathbf{g}^{*}, \mathbf{C}[G]\right)$. $H=\frac{1}{4}\left(\begin{array}{cc}1 & 0 \\ 0 & -1\end{array}\right), E_{+}=\frac{1}{2}\left(\begin{array}{ll}0 & 1 \\ 0 & 0\end{array}\right), E_{-}=\frac{1}{2}\left(\begin{array}{ll}0 & 0 \\ 1 & 0\end{array}\right)$ is a basis for $\mathbf{g}, L^{0}, L^{+}, L^{-}$the dual basis for $\mathbf{g}^{*}$. The action of $\mathbf{g}^{*}$ on $\mathbf{C}[G]$ as defined in Proposition (4.4) is as follows:

$$
\left\{\begin{array}{l}
\sigma\left(L^{0}\right)=-b c(N+2)-\left(b \frac{\partial}{\partial b}+c \frac{\partial}{\partial c}\right) \\
\sigma\left(L^{+}\right)=\frac{1}{2} a b(N+2)-b \frac{\partial}{\partial d} \\
\sigma\left(L^{-}\right)=-\frac{1}{2} c d(N+2)+c \frac{\partial}{\partial a}
\end{array}\right.
$$

where $N=a \frac{\partial}{\partial a}+b \frac{\partial}{\partial b}+c \frac{\partial}{\partial c}+d \frac{\partial}{\partial d}$.

We choose a basis for $\mathbf{C}[G]$ as follows

$$
X_{p, q, n}:=a^{p} b^{q}(b c)^{n}, \quad p, q \in \mathbb{Z}, \quad n \geqq 0,
$$

where $a^{p}:=d^{-p}, b^{q}:=c^{-q}$ if $p, q<0$. On this basis we get

$$
\sigma\left(L^{0}\right) X_{p, q, n}=-(|q|+2 n) X_{p, q, n}-f_{p, q, n} X_{p, q, n+1},
$$




$$
\begin{aligned}
& 2 \sigma\left(L^{+}\right) X_{p, q, n}=\left\{\begin{array}{lll}
f_{p, q, n} X_{p+1, q+1, n} & p \geqq 0, & q \geqq 0 \\
f_{p, q, n} X_{p+1, q+1, n+1} & p \geqq 0, & q<0 \\
f_{p, q, n} X_{p+1, q+1, n+1}+g_{p, q, n} X_{p+1, q+1, n} & p<0, & q \geqq 0 \\
f_{p, q, n} X_{p+1, q+1, n+2}+g_{p, q, n} X_{p+1, q+1, n+1} & p<0, & q<0
\end{array},\right. \\
& -2 \sigma\left(L_{-}\right) X_{p, q, n}=\left\{\begin{array}{lll}
f_{p, q, n} X_{p-1, q-1, n+2}+g_{p, q, n} X_{p-1, q-1, n+1} & p>0, & q>0 \\
f_{p, q, n} X_{p-1, q-1, n+1}+g_{p, q, n} X_{p-1, q-1, n} & p>0, & q \leqq 0 \\
f_{p, q, n} X_{p-1, q-1, n+1} & p \leqq 0, & q>0 \\
f_{p, q, n} X_{p-1, q-1, n} & p \leqq 0, & q \leqq 0
\end{array},\right.
\end{aligned}
$$

where $f_{p, q, n}=|p|+|q|+2 n+2, g_{p, q, n}=-|p|+|q|+2 n+2$.

Note

$$
\mathbf{C}[G]=\bigoplus_{\lambda \in \mathbb{Z}} V_{\lambda}, \quad V_{\lambda}=\bigoplus_{p-q=\lambda, n \geqq 0} \mathbf{C}\left[X_{p, q, n}\right],
$$

and $V_{\lambda}$ is invariant under the action of $\mathrm{g}^{*}$ as expected.

$H_{n}\left(\mathrm{~g}^{*}, V_{\lambda}\right)$ is computed by the double complex given in Lemma (4.11), and we choose to compute the $\mathbf{n}_{\mathbf{C}}$-homology first. Note $\left[L^{+}, L^{-}\right]=0$, this again can be computed by a double complex. The calculation is straightforward and we simply write down the result here.

Case 1. $\lambda=0$

$$
\begin{aligned}
& H_{n}\left(\mathbf{n}_{\mathbf{c}}, V_{0}\right)=0, \quad n>0, \\
& H_{0}\left(\mathbf{n}_{\mathbf{c}}, V_{0}\right)=\mathbf{C}[1] \oplus \mathbf{C}[b c] .
\end{aligned}
$$

$\mathbf{a}_{\mathbf{c}}$ acts on $H_{0}\left(\mathbf{n}_{\mathbf{c}}, V_{0}\right)$ non-semisimply,

$$
\begin{aligned}
\sigma\left(L^{0}\right) 1 & =-2 c \\
\sigma\left(L^{0}\right) b c & =-2 b c-4(b c)^{2}=-2 \sigma\left(L^{+}\right) d c \cong 0 .
\end{aligned}
$$

Therefore

$$
\begin{aligned}
& H_{0}\left(\mathbf{g}^{*}, V_{0}\right)=\mathbf{C}[1], \\
& H_{1}\left(\mathbf{g}^{*}, V_{0}\right)=\mathbf{C}\left[b c \otimes L^{0}+2 d c \otimes L^{+}\right] .
\end{aligned}
$$

Case 2. $\lambda=p-q>0$

$$
\begin{aligned}
& H_{1}\left(\mathbf{n}_{\mathbf{c}}, V_{\lambda}\right)=\mathbf{C}\left[a^{(\lambda / 2)+1} c^{(\lambda / 2)-1} \otimes L^{-}+a^{(\lambda / 2)-1} c^{(\lambda / 2)+1} \otimes L^{+}\right], \\
& H_{0}\left(\mathbf{n}_{\mathbf{c}}, V_{\lambda}\right)=\mathbf{C}\left[a^{\lambda}\right] \oplus \mathbf{C}\left[c^{\lambda}\right] \oplus \mathbf{C}\left[a^{\lambda / 2} c^{\lambda / 2}\right] .
\end{aligned}
$$

Of course terms involving $\frac{\lambda}{2}$ are there only when $\frac{\lambda}{2}$ makes sense, i.e., is an integer. Even when they exist, $\mathbf{a}_{\mathbf{c}}$ acts with non-zero eigenvalue. So they do not survive to be in the $\mathbf{g}^{*}$-homology. On the other hand

$$
\begin{aligned}
& \sigma\left(L^{0}\right) a^{\lambda}=-(\lambda+2) a^{\lambda}(b c)=-2 \sigma\left(L^{+}\right) a^{\lambda-1} c \cong 0 \\
& \sigma\left(L^{0}\right) c^{\lambda}=-\lambda c^{\lambda}-(\lambda+2) c^{\lambda}(b c)=2 \sigma\left(L_{-}\right) a c^{\lambda-1} \cong 0 .
\end{aligned}
$$


Thus

$$
\begin{aligned}
& H_{0}\left(\mathbf{g}^{*}, V_{\lambda}\right)=\mathbf{C}\left[a^{\lambda}\right] \oplus \mathbf{C}\left[c^{\lambda}\right], \\
& H_{1}\left(\mathbf{g}^{*}, V_{\lambda}\right)=\mathbf{C}\left[a^{\lambda} \otimes L^{0}+2 a^{\lambda-1} c \otimes L^{+}\right] \oplus \mathbf{C}\left[c^{\lambda} \oplus L^{0}-2 a c^{\lambda-1} \otimes L^{-}\right] .
\end{aligned}
$$

Case 3. $\lambda<0$ is similar to case 2 .

B.2. The Traces for the Quantum Algebra. We want to check that the linear functionals defined in Lemma (6.2.6) are indeed traces, i.e., they vanish on the commutators $\left[\mathrm{C}_{h}\left[S L_{2}\right], \mathbf{C}_{h}\left[S L_{2}\right]\right]$. By Proposition (2.4), the commutator of a Hopf algebra $A$ is the same as the image of $A$ under the right action of $A$ via $\rho$. So we only need to check that the linear functionals in Lemma (6.2.6) vanish on $\rho\left(t_{i j}\right)\left(\mathbf{C}_{h}\left[S L_{2}\right]\right)$, where $\left(t_{i j}\right)=\left(\begin{array}{ll}a & b \\ c & d\end{array}\right)$ are the generators of $\mathbf{C}_{h}\left[S L_{2}\right]$. Recall

$$
\rho\left(t_{i j}\right) f=\sum_{k} S\left(t_{k j}\right) f t_{i k},
$$

and

$$
S\left(\left(\begin{array}{ll}
a & b \\
c & d
\end{array}\right)\right)=\left(\begin{array}{cc}
d & -q b \\
-q^{-1} c & a
\end{array}\right),
$$

where we denote $q=e^{h}$. We have

$$
\left\{\begin{array}{l}
\rho(b)\left(a^{k} b^{m} c^{n}\right)=q^{-k}\left(1-q^{k+m+n+2}\right)(a b)\left(a^{k} b^{m} c^{n}\right) \\
\rho(b)\left(d^{k} b^{m} c^{n}\right)=\left(1-q^{m+n+2-k}\right) d^{k-1} b^{m+1} c^{n}+q^{1-2 k}\left(1-q^{k+m+n+2}\right) d^{k-1} b^{m+2} c^{n+1} \\
\rho(c)\left(a^{k} b^{m} c^{n}\right)=\left(1-q^{k-m-n-2}\right) a^{k-1} b^{m} c^{n+1}+q^{2 k-1}\left(1-q^{-k-m-n-2}\right) a^{k-1} b^{m+1} c^{n+2} \\
\rho(c)\left(d^{k} b^{m} c^{n}\right)=q^{k}\left(1-q^{-k-m-n-2}\right)(d c)\left(d^{k} b^{m} c^{n}\right) \\
\rho(a)\left(a^{k} b^{m} c^{n}\right)=q^{m+n} a^{k} b^{m} c^{n}-q^{k-1}\left(1-q^{k+m+n+2}\right) a^{k} b^{m+1} c^{n+1} \\
\rho(a)\left(d^{k} b^{m} c^{n}\right)=q^{m+n} d^{k} b^{m} c^{n}-q^{-k-1}\left(1-q^{k+m+n+2}\right) d^{k} b^{m+1} c^{n+1} \\
\rho(d)\left(a^{k} b^{m} c^{n}\right)=q^{-m-n} a^{k} b^{m} c^{n}-q^{k+1}\left(1-q^{-k-m-n-2}\right) a^{k} b^{m+1} c^{n+1} \\
\rho(d)\left(d^{k} b^{m} c^{n}\right)=q^{-m-n} d^{k} b^{m} c^{n}-q^{-k+1}\left(1-q^{-k-m-n-2}\right) d^{k} b^{m+1} c^{n+1} .
\end{array}\right.
$$

From this it is easy to see the tracial properties of the linear functionals in Lemma (6.2.6).

The family of traces $\tau_{n}^{e}$ is easy to understand. They correspond to the unquantized Cartan subgroup. How to understand the $\tau_{n}^{s}, n \neq 0$ ? The answer is that while $\tau_{n}^{e}$ corresponds to the point-like symplectic leaves, $\tau_{n}^{s}$ corresponds to the extended symplectic leaves in the Poisson Lie group $\left(S L_{2}, \pi\right)$. In [42] Soibelman and Vaksman construct irreducible modules for $C_{h}[S U(2)]$ corresponding to the symplectic leaves of the Poisson Lie group $S U(2)$. They work with the compact quantum group and we work with the complex quantum group. The difference is not important in the discussion here. For the trivial symplectic leaves corresponding to the Cartan subgroup, they get one-dimensional representations. This provides the family of traces $\tau_{n}^{e}$. For the non-trivial symplectic leaves, the corresponding Levendorski-Soibelman-Vaksman modules are infinite dimensional. Specifically, they are

$$
\mathscr{H}_{\alpha}=\left\{e_{0}, e_{1}, \ldots\right\}
$$


with the module structure given by

$$
d \cdot e_{0}=0, \quad a \cdot e_{n}=e_{n+1}, \quad b \cdot e_{n}=\alpha q^{n} e_{n}, \quad c \cdot e_{n}=\beta q^{n} e_{n},
$$

where $\alpha \beta=-q$. From this module we can get formally the following "trace," with the only non-zero value given by

$$
\tau_{\alpha}\left(b^{m} c^{n}\right)=\frac{\alpha^{m} \beta^{n}}{1-q^{m+n}}, \quad m, n \geqq 0 .
$$

But this is not really a trace on $\mathrm{C}_{h}\left[S L_{2}\right]$, since

$$
\tau_{\alpha}(a d-d a)=\left(q^{-1}-q\right) \tau_{\alpha}(b c)=-q^{-2} \neq 0 .
$$

This is due to the fact that $a, d$ are not of "trace class." The way out of this difficulty is to look at the weight decomposition of $\tau_{\alpha}$,

$$
\tau_{\alpha}=\sum_{n \in \mathbb{Z}} \alpha^{n} \tau_{n}^{s}
$$

We find that $\tau_{n}^{s}, n \neq 0$ are traces and $\tau_{0}^{s}$ is not a trace. As we have seen in Subsect. 6.2, this weight space decomposition proves to be very effective in the general case.

B.3. The Results. As we remarked in Subsect. 6.2, the construction of the traces in the previous subsection actually shows that the spectral sequence is degenerate at $E^{2}$ for $\mathbf{C}_{h}\left[S L_{2}\right]$. Thus

$$
H H_{n}\left(\mathbf{C}_{h}[G]\right) \cong H_{n}\left(\mathbf{g}^{*}, \mathbf{C}[G]\right) \otimes \mathbf{C}((h)) .
$$

As for the cyclic homology, the direct computation is actually not hard to carry out for quantum $\mathrm{SL}_{2}$. Corresponding to the new complex for the Hochschild homology (2.16), there is a new double complex $\left(C_{*}(A), d, D\right)$ for the cyclic homology, where $D=\xi^{\prime} B \xi$ just as $d=\xi^{\prime} b \xi$. We consider the spectral sequence associated with this new double complex whose $E^{1}$ term is the Hochschild homology. Since only $H H_{0}$ and $H H_{1}$ is non-zero, the double complex for the $E^{2}$ becomes

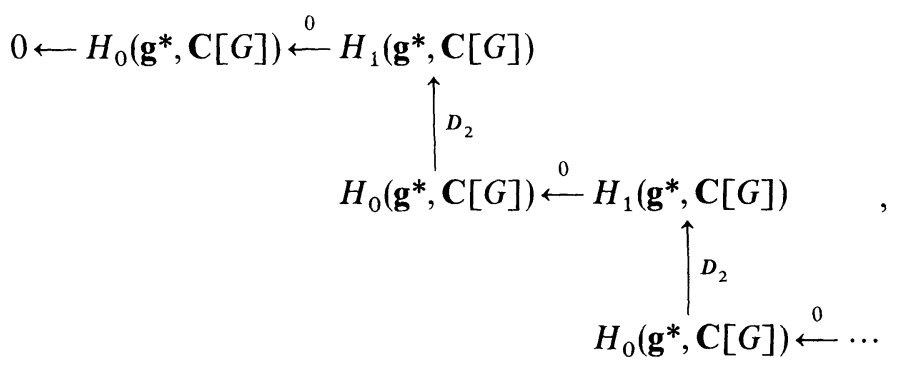

where $D_{2}(f)=\eta\left(\sum \tilde{f}_{j} \otimes f_{j}+f \otimes 1\right)=\sum \tilde{f}_{j} \otimes\left(d f_{j}\right)(e)$ with $\Delta(f)=\sum f_{j} \otimes \tilde{f}_{j}$.

It is easy to calculate

$$
\begin{aligned}
& D_{2}\left(a^{\lambda}\right)=\lambda\left(a^{\lambda} \otimes L^{0}+2 a^{\lambda-1} c \otimes L^{+}\right), \\
& D_{2}\left(c^{\lambda}\right)=\lambda\left(c^{\lambda} \otimes L^{0}+-2 a c^{\lambda-1} \otimes L^{-}\right) .
\end{aligned}
$$


Therefore we get

$$
H C_{n}\left(\mathbf{C}_{h}[G]\right) \cong \begin{cases}H_{0}\left(\mathbf{g}^{*}, \mathbf{C}[G]\right) \otimes \mathbf{C}((h)) & n=0 \\ H_{\mathrm{DR}}^{1}\left(T_{\mathbf{C}}\right) \otimes \mathbf{C}((h)) & n=1,3,5, \ldots \\ H_{\mathrm{DR}}^{0}\left(T_{\mathbf{C}}\right) \otimes \mathbf{C}((h)) & n=2,4,6, \ldots\end{cases}
$$

as expected.

\section{References}

1. Alvarez-Gaumé, L., Gomez, G., Sierra, G.: Quantum group interpretation of some conformal field theories. Phys. Lett. 220B, 142-152 (1989); Hidden quantum symmetrie's in rational conformal field theories. Nucl. Phys. B319, 155-186 (1989)

2. Brylinski, J.: Some examples of Hochschild and cyclic homology. In: Lecture Notes in Math. vol. 1271, pp. 33-72. Berlin, Heidelberg, New York: Springer 1987

3. Brylinski, J.: A differential complex for Poisson manifold. J. Diff. Goem. 28, 93-114 (1988)

4. Brylinski, J., Getzler, E.: The homology of algebras of pseudodifferential symbols and the non-commutative residue. $K$-theory $1,385-403$ (1987)

5. Casselman, W.: Jacquet modules for real reductive groups. In: Proceedings ICM, Helsinki, 1978

6. Casselman, W., Osborne, M. S.: The n-cohomology of representations with an infinitesimal character. Compositio Math. 31, 219-227 (1975)

7. Casselman, W., Osborne, M. S.: The restriction of admissible representations to n. Math. Ann. 233, 193-198 (1978)

8. Connes, A.: Non-commutative differential geometry. Publ. Math. IHES 62, 257-360 (1985)

9. Connes, A.: Essay on physics and non-commutative differential geometry. In: The Interface of mathematics and particle physics. Quillen, D. G., Segal, G. B., Tsou, S. T., (eds.), Oxford: Oxford University Press 1990

10. Daletskiy, Yu. L., Tsygan, B. L.: Hamiltonian operator and Hochschild homology. Funct. Anal. Appl. 19, (4) 82-83 (1985)

11. Drinfeld, V. G.: Quantum groups. In: Proceedings ICM, Berkeley, 1986

12. Drinfeld, V. G.: Hamiltonian structure on Lie groups, Lie bialgebras and the geometric meaning of the classical Yang-Baxter equation. Sov. Math. Dokl. 27, 68-71 (1982)

13. Feigin, B. L., Tsygan, B. L.: Lie algebra homology of generalized Jacobi matrices. Funct. Anal. Appl. 17, no. (2) 86-87 (1983)

14. Feigin, B. I., Tsygan, B. I.: Additive $K$-theory. In: Lecture Notes in Math. vol. 1289, pp. 67-209. Berlin, Heidelberg, New York: Springer 1988

15. Feigin, B. I., Tsygan, B. I.: Cyclic homology of algebras with quadratic relations, universal enveloping algebras and group algebras. In: Lecture Notes in Math. vol. 1289, pp. 210-239. Berlin, Heidelberg, New York: Springer 1988

16. Feigin, B. L., Tsygan, B. L.: Riemann-Roch theorem and Lie algebra cohomology. In: Proceedings of Second Winter School at Crni, Rend. Math. Palermo (1989)

17. Goodwillie, T.: Cyclic homology, derivations and free loop space. Topology 24, 187-216 (1985)

18. Hecht, H., Schmid, W.: Characters, asymptotics and $\mathbf{n}$-homology of Harish-Chandra modules. Acta. Math. 151, 49-151 (1983)

19. Helgason, S.: Differential geometry, Lie groups, and symmetric spaces. New York:, London: Academic Press 1978

20. Hilton, P. J., Stammbach, U.: A course in homological algebra. Berlin, Heidelberg, New York: Springer 1970

21. Jimbo, M.: A $q$-difference analogue of $U(\mathbf{g})$ and Yang-Baxter equation. Lett. Math. Phys. 10, 63-69 (1985)

22. Kassel, C.: L'homologie cyclique des algebres enveloppantes. Invent. Math. 91, 221-251 (1988)

23. Kirillov, A. N., Reshetikhin, N. Yu.: Representations of the algebra $U_{q}\left(s l_{2}\right), q$-orthogonal polynomials and invariants of links. LOMI-Preprint E-9-88, 1988 
24. Kirllov, A. N., Reshetikhin, N. Yu.: $q$-Weyl group and a multiplicative formula for $R$-matrix. Commun. Math. Phys. 134, 421-431 (1991)

25. Knapp, A. W.: Representation theory of semisimple groups. Princeton, NJ: Princeton University Press 1986

26. Levendorski, S., Soibelman, Ya.: Algebras of functions on compact quantum groups, Schubert cells and quantum tori, Preprint

27. Loday, J.-L., Quillen, D.: Cyclic homology and Lie algebra homology of matrices. Comment. Math. Helv. 59, 565-591 (1984)

28. Lu, J.-H., Weinstein, A.: Poisson Lie groups, dressing transformations and the Bruhat decomposition. J. Diff. Geom. 31, 501-526 (1990)

29. Lusztig, G.: Quantum groups at roots of 1, to appear in Geom. Ded. (1990)

30. Mack, G., Schomerus, V.: Conformal field algebras with quantum symmetry from the theory of superselection sectors. Commun. Math. Phys. 134, 139-196 (1990)

31. Manin, Yu. I.: Quantum groups and non-commutative geometry. Centre de Recherches Mathematiques, Universite de Montreal, 1988

32. Masuda, T., Natsume, T.: Cyclic cohomology of certain affine schemes. Publ. RIMS 21, 1261-1279 (1985)

33. Masuda, T., Nakagami, Y., Watanabe, J.: Non-commutative differential geometry on the quantum $S U(2), I, K$-theory 4, 157-180 (1990)

34. Moore, G., Seiberg, N.: Naturality in conformal field theory. Nucl. Phys. B313, 16-40 (1989); Classical and quantum conformal field theory. Commun. Math. Phys. 123, 177-254 (1989)

35. Parshall, B., Wang, J.-P.: Quantum linear groups. Memoirs of AMS, no. 439

36. Rosenberg, A.: Private communications

37. Rosso, M.: An analogue of P.B.W. theorem and the universal $R$-matrix for $U_{h}(s l(N+1))$. Commun. Math. Phys. 124, 307-718 (1989)

38. Schmid, W.: Vanishing theorems for Lie algebra cohomology and the cohomology of the discrete subgroups of semisimple Lie groups. Adv. Math. 41, 78-113 (1981)

39. Semenov-: ian-Shansky, M.: Dressing transformation and Poisson group actions. Publ. RIMS 21, 1237-1260 (1985)

40. Serre, J.-P.: Algebra locale, multiplicites: cours an College de France, 1957-1958. Lecture Notes in Math. vol. 11. Berlin, Heidelberg, New York: Springer 1975

41. Soibelman, Ya. S.: Irreducible representations of the function algebra on the quantum group $S U(n)$ and Schubert cells, Sov. Math. Dokl. 40, 34-38 (1990)

42. Soibelman, Y., Vaksman, L.: Algebra of functions on the quantum group SU(2). Funct. Anal. Appl. 22 (3), 1-14 (1988)

43. Takhtajan, L. A.: Non-commutative homology of quantum tori. Funct. Anal. Appl. 23, 147-149 (1989)

44. Tsygan, B. L.: Homology of matrix Lie algebras over rings and Hochschild homology. Usp. Mat. Nauk. 38, (2) 217-218 (1983)

45. Warner, G.: Harmonic Analysis on Semi-simple Lie Groups, Vol. I. Berlin, Heidelberg, New York: Springer 1972

46. Wodzicki, M.: Cyclic homology of pseudodifferential operators and noncommutative Euler class. C. R. Acad. Sci. Paris Ser. I Math. 306, 321-325 (1988) 
